\title{
Diameters of Spherical Alexandrov Spaces and Curvature One Orbifolds
}

\author{
SARAH J. GREENWALD
}

\begin{abstract}
Let $G$ be a closed, non-transitive subgroup of $O(n+1)$, where $n \geq 2$, and let $Q^{n}=S^{n} / G$. We will show that for each $n$ there is a lower bound for the diameter of $Q^{n}$. If $G$ is finite, then $Q^{n}$ is an orbifold of constant curvature one, and an explicit lower bound can be given. For Coxeter groups, the resulting lower bound is independent of dimension. Otherwise, $Q^{n}$ is a spherical Alexandrov space and we will show existence of a lower bound. In the process, we will compute some examples of quotient spaces and their diameters.
\end{abstract}

\section{INTRODUCTION}

While representations of compact Lie groups are well understood, the geometry of the corresponding spherical quotients is virtually unknown. Let $G$ be a closed, non-transitive subgroup of $O(n+1)$, where $n \geq 2$, and let $Q^{n}=S^{n} / G$. The goal of this paper is to find lower bounds for the diameter of $Q^{n}$.

Knowing how small the diameter can get not only gives information about $Q^{n}$ itself, but can also lead to other interesting results. For example, for the equivariant sphere theorem in [21], let $G$ be a closed subgroup of the isometries of a closed manifold $M$ with positive sectional curvature. Given any point $p$ on the manifold, the tangent space to the orbit $G p$ at $p$ is invariant under the isotropy group at $p$, which is a subgroup of the orthogonal group, $O\left(T_{p} M\right)$. Hence, the normal space is also left invariant. Let $S_{[p]}$ be the quotient of the unit sphere in this normal space by the isotropy group at $p$. This is one of the above spaces $Q^{n}$. If two points $p$ and $q$ can be found on $M$ so that the diameters of $S_{[p]}$ and $S_{[q]}$ are both less than $\pi / 4$, then $M$ is the union of tubular neighborhoods about the orbits of these points. Thus, local diameter information gives global results about the structure of the manifold. 
For lower bounds on the diameter, it is only necessary to examine irreducible actions, since the diameter of a reducible action is $\pi$ when there is a point of $S^{n}$ fixed by the entire group, and $\pi / 2$ otherwise (see [21] and [4]).

If the group is finite, then the resulting quotient space is a constant curvature one orbifold. If $\Gamma$ acts properly discontinuously and freely, then $S^{n} / \Gamma$ is a manifold. All such groups are classified in [34]. McGowan [25] used this classification to show that diameters of these manifolds are bounded below by $\frac{1}{2} \arccos (\tan (3 \pi / 10) / \sqrt{3})$, which is approximately $\pi / 9.63$ (see also [18]). This lower bound is optimal and occurs in dimension $n=3$.

The problem of classifying all finite subgroups of $O(n+1)$ is equivalent to classifying all orthogonal representations of finite groups. Hence, methods of exhaustive computation, as in the manifold case, are not feasible beyond a few small dimensions.

After providing the necessary background in Section 2, this paper discusses lower bounds on the diameter of $S^{n} / \Gamma$ when $\Gamma$ is closed and non-transitive, applications, and an exhaustive computation of the resulting spaces and their diameters for a wide class of groups.

We first examine the diameters of spaces resulting from finite groups in Section 3.

Theorem $A$. If $\Gamma$ is finite, then there exists $\varepsilon$, depending only on $n$, so that $\operatorname{diam}\left(S^{n} / \Gamma\right) \geq \varepsilon(n)$.

The lower bound $\varepsilon(n)$ is explicit in the proof of the theorem, although it does tend to 0 as $n$ gets large. A brief sketch of the proof follows. Using ideas in the proof of Bieberbach's first theorem [9], it can be shown that elements within a neighborhood of the identity commute, since $\Gamma$ is a finite group. Then, a finite index abelian subgroup of $\Gamma$ can be found so that the index can be universally controlled. The diameter of the abelian subgroup, which we prove to be at least $\pi / 2$, and the index are used to bound the diameter of $S^{n} / \Gamma$ by a constant which depends only on $n$.

Corollary A. If $\Gamma$ is a finite subgroup of the isometry group of $M^{n}$, where $M^{n}$ is either $\mathbb{C} P^{n}$ or $\mathbb{U} P^{n}$, then there exists $\varepsilon$ depending only on $n$, so that $\operatorname{diam}\left(M^{n} / \Gamma\right) \geq \varepsilon(n)$.

The proof is an extension of Theorem 3.14 via a Hopf-fibration argument.

Theorem B. If $\Gamma$ is a Coxeter group, generated by reflections, then $\operatorname{diam}\left(S^{n} / \Gamma\right) \geq \pi / 8.10$.

This is achieved as the diameter of a quotient of $S^{3}$. The diameter of Coxeter orbifolds, resulting from groups generated by reflections, increases monotonically in $n$, and as $n$ approaches infinity, the diameter approaches $\pi / 2$. 
We next examine the diameters of spaces resulting from closed, infinite, nontransitive groups in Section 4. If the group is infinite, then the resulting quotient space is an Alexandrov space with curvature bounded below. The explicit orbifold lower bounds in Theorem A do not apply, since discreteness was needed in the proof. When the quotient space is an interval, the action is called a cohomogeneity-one action. Since the orbits $\Gamma p$ are isoparametric hypersurfaces in spheres, the length of the intervals are at least $\pi / 6$ (see [23]). There are only two examples, in dimensions 7 and 13, where the diameter is equal to $\pi / 6$. However, unlike the manifold and Coxeter orbifold cases, there is an entire class of cohomogeneity-one actions on $S^{n}$, including actions for arbitrarily large dimensions, which result in a quotient space of diameter $\pi / 4$.

Theorem $C$. If $\Gamma$ is a non-transitive group, then there exists $\varepsilon$, depending only on $n$, so that $\operatorname{diam}\left(S^{n} / \Gamma\right) \geq \varepsilon(n)$.

The lower bounds are not explicit since the proof is by contradiction. We show that a non-transitive sequence of groups cannot converge to a transitive subgroup of $S O(n+1)$. The proof relies on a theorem of Montgomery and Zippin [28], which says that groups converging to a Lie group must eventually be conjugate to subgroups. Corollary 3.14.1 is needed when the transitive group is not simple.

Finally, we summarize the diameter results in Table 2. For quotient space orbifolds, we find lower bounds which decrease to 0 as $n$ gets large, but it is an interesting question whether optimal lower bounds actually do decrease with $n$, or whether the optimal lower bound is always achieved in dimension 3 , as in the manifold and Coxeter orbifold cases, and whether it is always true, as in the manifold and Coxeter orbifold cases, that the optimal lower bound for the diameter increases to $\pi / 2$ as $n$ goes to infinity.

I would like to thank my advisor, Wolfgang Ziller, for his guidance and patience. I am grateful to Karsten Grove, whose idea of taking limits of groups was the beginning of the proof of Theorem 4.3, and Kevin Whyte, for the idea of the proof of Theorem 3.14.

\section{BACKGROUND}

2.1. Riemannian orbifolds. We present some basic ideas and intuition about Riemannian orbifolds along with references where rigorous definitions and proofs can be found.

While a manifold locally looks like the Euclidean space $\mathbb{R}^{n}[13]$, an orbifold locally looks like the quotient of $\mathbb{R}^{n}$ by a discrete group action (see [29, p. 662] or [30, Section 2]).

A Riemannian orbifold locally looks (isometrically) like the quotient of a Riemannian manifold by a finite subgroup of its isometry group [4, pp. 9-12]. See [4, pp. 24-28] and [20, Chapter 3] for examples of Riemannian orbifolds. 
Remark. In general, an orbifold is not even homeomorphic to a manifold. For example, look at $x \rightarrow-x$ as a $\mathbb{Z}_{2}$ action on $\mathbb{R}^{3}$. Now, $\mathbb{R}^{3} / \mathbb{Z}_{2}$ is homeomorphic to a cone on $\mathbb{R} P^{2}$, but is not homeomorphic to a manifold at the cone point. However, in dimension 2, any orbifold is homeomorphic to a manifold [30, p. 422]. Yet, orbifolds with cone points are not isomorphic or isometric to manifolds.

Remark. For the purpose of this paper, we drop the Riemannian label and assume that all of our orbifolds are Riemannian.

One can measure distance locally on an orbifold via lifting up to the Riemannian manifold to compute lengths. To measure distance globally, we add up the local lengths. While these local lifts are not unique, the length of a curve is well defined [4, pp. 18-22]. One can define the orbifold curvature as the curvature of the Riemannian manifold in the local lift. Other Riemannian geometric concepts can also be extended to orbifolds. For example, Toponogov's Theorem, Volume Comparison, Sphere Theorems, Finiteness Theorems and The Closed Geodesic Problem are all discussed in [4].

A good orbifold is the global quotient of a Riemannian manifold by a discrete subgroup of its isometry group [4, p. 11]. A bad orbifold is an orbifold which does not arise in this manner.

Lemma 2.1. ([32]) There are no bad constant curvature orbifolds.

Idea of proof. One can construct a developing map into the constant curvature space form $M=S^{n}, \mathbb{R}^{n}$, or $\mathbb{t}^{n}$ from the orbifold universal covering, using the fact that any of the local charts that are used to define constant curvature orbifolds are restrictions of global actions. (See [29, Chapter 13] for definitions of the developing map and orbifold universal cover).

Remark. Let $\Gamma \subset O(n+1)$ be finite. Then $O^{n}=S^{n} / \Gamma$ is a constant curvature one orbifold.

2.2. Spherical Alexandrov spaces. If $G$ is not a discrete group, but is instead a closed infinite group, then $S^{n} / G$ is a spherical Alexandrov space with curvature bounded below. This is a length space, where Riemannian notions such as distance and curvature are obtained by comparison with $S^{n}$ via Toponogov $[8,31]$.

\subsection{Reducible orthogonal actions and the diameter.}

Lemma 2.2. ([5], [26]) Let $O^{n}=S^{n} / G$, where $G \subset O(n+1)$ is closed. Then, $\operatorname{diam}(O)>\pi / 2$ iff $\operatorname{diam}(O)=\pi$ iff there is a point of $S^{n}$ fixed by the whole group.

Lemma 2.3. ([5], [26]) Let $O^{n}=S^{n} / G$, where $G \subset O(n+1)$ is closed. Then, $G$ is a reducible action iff $\operatorname{diam}(O)=\pi / 2$ or $\pi$.

Reducible actions lead to a large diameter of at least $\pi / 2$ by the above lemmas. In addition, the resulting diameter corresponding to a group is no larger than the resulting diameter corresponding to its subgroups [26]. 


\section{FINITE GROUPS}

3.1. Intuition and examples. As we saw in Section 2.3 , it is only necessary to examine irreducible actions for a lower bound on the diameter, since the diameter is $\pi$ when there is a point of $S^{n}$ fixed by the entire group, and $\pi / 2$ for all other reducible actions.

Orbifolds. If the group $G$ is finite, then the resulting quotient space is a constant curvature one orbifold. In order to obtain a small diameter, a first guess would be to look at the resulting space corresponding to a large group action.

\section{Example 3.1. Footballs in dimension 2}

Let $r=\left(\begin{array}{cc}r_{9} & 0 \\ 0 & 1\end{array}\right)$ be a $3 \times 3$ real matrix, where $r_{9}$ is a $2 \times 2$ rotation matrix with rotation angle $\pi \cdot q / p$, a rational multiple of $\pi$, and $p$ and $q$ are relatively prime. Then $S^{2} /\langle r\rangle=S^{2} / \mathbb{Z}_{2 p}$ is a football of diameter $\pi$, with isotropy $\mathbb{Z}_{2 p}$ at the cone points. By taking smaller rotations, we obtain thinner footballs. The quotient of $S^{2}$ by $S^{1}$, an infinite group, is a longitude of length $\pi$.

\section{Example 3.2. Orbifold lens spaces in dimension 3}

Let $\Gamma=\mathbb{Z}_{p}$ be generated by $\left(z_{1}, z_{2}\right) \rightarrow\left(e^{2 \pi i / p} z_{1}, e^{2 \pi i q / p} z_{2}\right)$, where $p$ and $q$ are integers and $z_{1}$ and $z_{2}$ are complex numbers. Let $L(3, p, q)=S^{3} / \Gamma$. This is a (manifold) lens space when $p$ and $q$ are relatively prime. Otherwise, $\Gamma$ does not act freely on $S^{3}$, and so $L(3, p, q)$ is an orbifold lens space. Since the $z_{1}$ complex plane is left invariant by $\Gamma$, we know that $\operatorname{diam}(L(p, q))$ is $\pi / 2$ or $\pi$. The diameter is $\pi / 2$ exactly when $q$ is not an integer multiple of $p$, since there is no point of $S^{3}$ fixed by the entire group.

We now look at abelian groups, which provide useful intuition and are necessary in the proof of Theorem 3.14.

Example 3.3. $S^{n} /$ maximal torus in $S O(n+1)$

Let $T(n+1)$ be the maximal torus in $S O(n+1)$.

- For $n$ even, $\operatorname{diam}\left(S^{n} / T(n+1)\right)=\pi$.

- For $n$ odd, $\operatorname{diam}\left(S^{n} / T(n+1)\right)=\pi / 2$.

For $n$ odd, the maximal torus $T(n+1)$ consists of all $n+1$ by $n+1$ real matrices of the form

$$
\left(\begin{array}{cccc}
r_{\vartheta_{1}} & 0 & \ldots & 0 \\
0 & r_{\vartheta_{2}} & \ldots & 0 \\
\vdots & \vdots & \ddots & \vdots \\
0 & 0 & 0 & r_{9_{(n+1) / 2}}
\end{array}\right)
$$

(see Example 3.1). Then, $S^{n} / T(n+1)$ has diameter $\pi / 2$ since there is no point of $S^{n}$ fixed by the entire group, but there are invariant subspaces. For $n=3$, $S^{3} / T(4)$ is an arc of length $\pi / 2$. For larger, odd $n$, the quotient space 
$S^{n} / T(n+1)$ consists of a spherical polyhedron with $(n+1) / 2$ vertices, where each edge has length $\pi / 2$.

For $n$ even, the maximal torus $T(n+1)$ consists of all matrices of the form

$$
\left(\begin{array}{cccc}
r_{\vartheta_{1}} & 0 & \ldots & 0 \\
0 & r_{\vartheta_{2}} & \ldots & 0 \\
\vdots & \vdots & \ddots & \vdots \\
0 & 0 & 0 & r_{\vartheta_{n / 2}} \\
0 & 0 & 0 & 1
\end{array}\right) .
$$

Then, $S^{n} / T(n+1)$ has diameter $\pi$ since the last coordinate, $x_{n+1}$, is fixed by the entire group. For $n=2$, as in Example 3.1, $S^{2} / T(3)$ is an arc of length $\pi$. For larger, even $n$, the quotient space $S^{n} / T(n+1)$ consists of a suspension of a polyhedron. Arcs of length $\pi$, which intersect at $(0,0, \ldots, 1)$ and $(0,0, \ldots,-1)$, are all $\pi / 2$ away from each other.

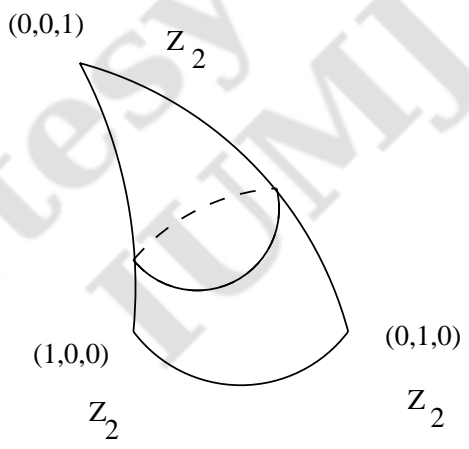

FIGURE $1 . S^{2} / \mathbb{Z}_{2} \times \mathbb{Z}_{2}$

The following is an example of a group which is abelian, but not contained in a maximal torus.

Example 3.4. The orbifold resulting from the group consisting of

$$
\left(\begin{array}{rrr}
1 & 0 & 0 \\
0 & -1 & 0 \\
0 & 0 & -1
\end{array}\right), \quad\left(\begin{array}{rrr}
-1 & 0 & 0 \\
0 & 1 & 0 \\
0 & 0 & -1
\end{array}\right), \quad\left(\begin{array}{rrr}
-1 & 0 & 0 \\
0 & -1 & 0 \\
0 & 0 & 1
\end{array}\right), \quad\left(\begin{array}{lll}
1 & 0 & 0 \\
0 & 1 & 0 \\
0 & 0 & 1
\end{array}\right)
$$

Notice this group is $\mathbb{Z}_{2} \times \mathbb{Z}_{2}$. It is abelian and is not contained in a maximal torus $S^{1}$ of $S O(3)$. The $x$-axis is invariant under this action, but not fixed, so the action is reducible with resulting diameter $\pi / 2$. A fundamental domain for the 
action is the orange peel wedge formed by half of the northern hemisphere, containing positive $x$ and $z$. On the boundary, $(0, y, z)$ and $(0,-y, z)$ are identified, and $(x, y, 0)$ and $(x,-y, 0)$ are identified. The resulting orbifold is a 3-corner pillow with $\mathbb{Z}_{2}$ isotropy at all three points.

The following is an example of an irreducible action, which we know will have resulting diameter less than $\pi / 2$ by Section 2.3 .

\section{Example 3.5. Three-cornered pillow shaped orbifold in dimension 2}

The icosahedral group $I$ acts on $S^{2}$ giving rise to $S^{2} / I$, a three-cornered pillow with isotropy labeled below and diameter $\arccos (\tan (3 \pi / 10) / \sqrt{3})$, which is approximately $\pi / 4.82$ ([15], [20]).

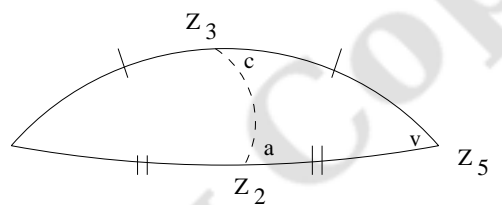

FIgURE 2. Icosahedral Fundamental Domain

Manifolds and the diameter. If $\Gamma$ acts properly discontinuously and freely, then $S^{n} / \Gamma$ is a manifold. McGowan [25] has shown that diameters of these manifolds are bounded below by $\frac{1}{2} \arccos (\tan (3 \pi / 10) / \sqrt{3})$, which is approximately $\pi / 9.63$ (see also [18]). This lower bound is optimal and occurs in dimension $n=3$, as follows.

First, note that a cyclic group $\mathbb{Z} u$ acts on $S^{2}$ by fixing an axis of rotation, as in Example 3.1. The icosahedral group $I$ also acts on $S^{2}$ as in Example 3.5. Pull back the group $\mathbb{Z}_{u} \times I$ in $S O(3) \times S O(3)$ into $S U(2) \times S U(2)$ to get $\mathbb{Z}_{2 u} \times I^{*}$. This acts on $S^{3}$ by quaternionic left and right multiplication. $S^{3} / \mathbb{Z}_{2 u} \times I^{*}$ is the desired manifold, which has the above diameter bound (compare with Section 4.1).

The diameters of all other manifolds $S^{n} / \Gamma$ are computed exhaustively and found to be larger than this manifold. The diameters increase monotonically in $n$ and approach $\pi / 2$ as $n$ approaches infinity.

3.2. Irreducible group condition. In this section we prove that a finite, irreducible group must satisfy a certain condition, which is independent of the dimension. In the process, we will prove some lemmas necessary for the next section. Notice that Lemmas 3.6 and 3.8 are true for any closed group, while finiteness is necessary in Lemma 3.9 and Theorem 3.11.

Lemma 3.6. Let $G \subset O(n+1)$ be closed. If $G$ is abelian, then the action is reducible and so $\operatorname{diam}\left(S^{n} / G\right)$ is $\pi / 2$ or $\pi$. 
Proof. After complexifying, $G$ acts as a commuting set of unitary operators, and hence we can diagonalize all the matrices simultaneously over $\mathbb{C}$ via an orthonormal basis of eigenvectors. Let $v_{i}$ be this orthonormal basis. Then, if $g \in G$, we know that $g v_{i}=e^{i 9} v_{i}$. If $e^{i 9}$ is real, then $v_{i}$ generates a real invariant subspace. Otherwise, we also have $g \bar{v}_{i}=e^{-i 9} \bar{v}_{i}$. Look at $\left\{v_{i}, \bar{v}_{i}\right\}=W \subset \mathbb{C}^{n+1}$ and $W \cap \mathbb{R}^{n+1}$. Now $G\left(W \cap \mathbb{R}^{n+1}\right) \subset W \cap \mathbb{R}^{n+1}$. Also, $v_{1}+\bar{v}_{1}, i\left(v_{1}-\bar{v}_{1}\right) \in W$, and so $W$ is a non-empty invariant subspace. Hence, $G$ is reducible.

Definition 3.7. In order to examine a neighborhood of the identity in $O(n+1)$, look at the sup norm on $G L(n+1),\|M\|=\sup \left\{|M x|: x \in \mathbb{R}^{n+1}\right.$ and

$|x|=1\}$, where $|x|$ is the Euclidean norm. Then define an epsilon ball about $M$ in $O(n+1)$ as $B_{\varepsilon}(\mathrm{Id})=\{M \in O(n+1):\|\mathrm{Id}-M\|<\varepsilon\}$.

Lemma 3.8. Consider $U=B_{1 / 2}(\mathrm{Id})$. This satisfies [9]:

(1) Let $g \in O(n+1), h \in U,[g,[g, h]]=\mathrm{id} \Rightarrow[g, h]=$ id.

(2) $g, h \in U \Rightarrow$ the sequence $g_{0}=g, g_{1}=[g, h], g_{2}=[g,[g, h]]=\left[g, g_{1}\right]$, $\ldots, g_{n}, \ldots$ converges to id $\in O(n+1)$.

In [9], this lemma is only proven for $U=B_{1 / 4}$ (Id), but one can easily modify the proof to work for $U=B_{1 / 2-\varepsilon}$ (Id), for any $\varepsilon>0$. To show that this holds for $U=B_{1 / 2}$ (Id), it is necessary to show that (2) holds. Assume for contradiction that (2) does not hold for $U=B_{1 / 2}$ (Id). Choose $g, h \in U$ which violate (2). Now, $g$, $h$ must be in $B_{1 / 2-\varepsilon}$ (Id) for some $\varepsilon>0$, and so (2) does not hold for $B_{1 / 2-\varepsilon}$ (Id), a contradiction.

Lemma 3.9. Let $\Gamma$ be a finite group in $O(n+1)$. If $\Gamma \subset U=B_{1 / 2}(\mathrm{Id})$, then $\Gamma$ is abelian.

Proof. Let $g, h \in \Gamma$. We know that $\Gamma$ is discrete since $\Gamma$ is finite. Since $g_{i} \rightarrow$ id in Lemma 3.8, then $g_{i}=$ id for some $i$. Applying (1) from the lemma repeatedly, we see that $g_{1}=[g, h]=\mathrm{id}$. Thus, $\Gamma$ is abelian.

Definition 3.10. Let $p \in S^{n}$ be a point with a trivial isotropy group for a discrete action of $\Gamma$ on $S^{n}$ (such points exist as in [22, p. 28]). A Dirichlet fundamental domain centered at $p$ is the set $\left\{x \in S^{n} \mid d(x, p) \leq d(x, g p)\right.$, $\forall g \in \Gamma\}$. Since $\Gamma$ is discrete, it is finite, so label the elements as $\gamma_{1}, \ldots, \gamma_{m}$. Let $H_{\gamma_{i}}=\left\{x \in S^{n} \mid d(x, p) \leq d\left(x, \gamma_{i}(p)\right\}\right.$. Then, the Dirichlet fundamental domain is $\bigcap H_{\gamma_{i}}$, which is a fundamental domain for the action of $\Gamma$ on $S^{n}$ [22, pp. 29-30].

Theorem 3.11. Given a Dirichlet fundamental domain, each co-dimension one face on the boundary arises from the half-space between the center point and its image under a group element. If the action is irreducible and finite, then one of these domain generating group elements moves some point $y$ in $S^{n}$ at least $\arccos \frac{7}{8}$, which is approximately $\pi / 6.2$, away from $y$. 
Proof. Let $\Gamma$ be finite, and let the action of $\Gamma$ be irreducible. Then, looking at the sup norm on $O(n+1)$, we will show that $\left\|\mathrm{Id}-g_{i}\right\| \geq \frac{1}{2}$ for some $i$, where $g_{i}$ generates the Dirichlet fundamental domain.

Assume not for contradiction. Then all the $g_{i}$ are in $B_{1 / 2}(\mathrm{Id})$, the ball of radius $\frac{1}{2}$ about the identity. Applying Lemma 3.9, we see that all the $g_{i}$ commute. Since these generate the group, then the entire group is abelian. By Lemma 3.6, the action is reducible, a contradiction. Thus, by definition of the sup norm, the Euclidean distance between $y$ and $g_{i}(y)$ must be greater than or equal to $\frac{1}{2}$ for some $i$ and for some $y \in S^{n}$. For any such $i$ and $y$, form the triangle consisting of the vectors $y$ and $g_{i}(y)$ through the point $(0, \ldots, 0)$ and angle $\vartheta$ between them, and with $c$ as the side opposite the angle $\vartheta$. Using trigonometry, we see that $\frac{1}{4} \leq c^{2}=2-2 \cos \vartheta$ or $2 \cos \vartheta \leq \frac{7}{4}$. Hence, $\vartheta \leq \arccos \frac{7}{8}$. Converting back to spherical distance, which is the Euclidean angle between vectors, we see that $d(y, i(y)) \geq \arccos \frac{7}{8}$, as desired.

Remark 3.12. This condition on the group is independent of the dimension.

3.3. Explicit lower bound given a fixed dimension. In this section, we will prove that given a fixed dimension, there exists a lower bound on the diameter resulting from finite groups. This lower bound is explicit and depends only on the dimension. While finiteness is used in the proof of Theorem 3.14, notice that Lemma 3.13 holds for any closed, non-transitive group $G \subset O(n+1)$.

Lemma 3.13. Let $G \subset O(n+1)$ be closed and non-transitive. If $G^{\prime}$ has index $k$ in $G$, then $\operatorname{diam}\left(S^{n} / G\right) \geq \operatorname{diam}\left(S^{n} / G^{\prime}\right) / 2(k-1)$ (compare with [18, p. 103]).

Proof. Let $d$ be the diameter of $S^{n} / G$ and let $p \in S^{n} / G$. Now $B_{d}(p)$, the ball of radius $d$ about $p$, must cover $S^{n} / G$. Since the index of $G^{\prime}$ in $G$ is $k$, notice that $S^{n} / G$ is a k-fold cover of $S^{n} / G^{\prime}$ and so $p$ must lift to $p_{1}^{\prime}, \ldots, p_{k}^{\prime}$ via the coset map. Hence $B_{d}(p)$ must lift to $B_{d}\left(p_{1}^{\prime}\right), B_{d}\left(p_{2}^{\prime}\right), \ldots, B_{d}\left(p_{k}^{\prime}\right)$, where $p_{i}^{\prime} \in S^{n} / G^{\prime}$. These balls cover $S^{n} / G^{\prime}$. Let $p^{\prime}$ be in the intersection of any two balls in $S^{n} / G^{\prime}$ and let $q^{\prime} \in S^{n} / G^{\prime}$. To show that $d\left(p^{\prime}, q^{\prime}\right) \leq 2(k-1) d$, create a path from $p^{\prime}$ to $q^{\prime}$ by traveling through the $p_{i}^{\prime} s$, the centers of the balls.

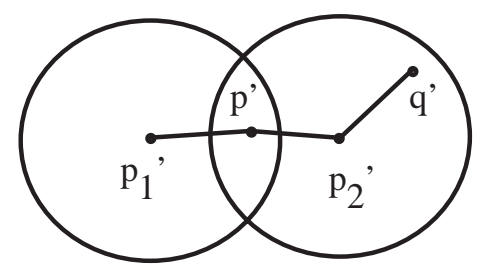

FIGURE 3. Index 2 
The length of this path is at most $2(k-1) d$, since if you hit any ball more than once, you will obtain a loop, which can be discarded. Notice that $p$ was arbitrary, so that given any $p^{\prime} \in S^{n} / G^{\prime}$, we can find some point in $S^{n} / G$ and repeat the process of lifting so that $p^{\prime}$ arises in the intersection of two balls as above. Hence $d\left(p^{\prime}, q^{\prime}\right) \leq 2(k-1) d$ for any points $p^{\prime}, q^{\prime} \in S^{n} / G^{\prime}$, and so $\operatorname{diam}\left(S^{n} / G^{\prime}\right) \leq 2(k-1) d$, as desired.

Corollary 3.13.1. If $G^{\prime} \in S O(n+1)$ with $\operatorname{diam}\left(S^{n} / G^{\prime}\right)=d^{\prime}$ and $G \in$ $O(n+1)$ is a 2-fold extension of $G^{\prime}$, then $\operatorname{diam}\left(S^{n} / G\right) \geq d^{\prime} / 2$.

Theorem 3.14. Let $\Gamma \subset O(n+1)$ be finite. There exists $\varepsilon$ depending only on $n$ so that $\operatorname{diam}\left(S^{n} / \Gamma\right) \geq \varepsilon(n)$.

Proof. Let $\Gamma \subset O(n+1)$ be finite. Let $\Gamma^{\prime}$ be the subgroup generated by $\Gamma \cap U \subset B_{1 / 2}$ (Id), which is abelian by Lemma 3.6.

We will universally bound the index of $\Gamma^{\prime}$ in $\Gamma$ with a constant depending only on the dimension $n$. Write $\Gamma=\delta_{1} \Gamma^{\prime} \cup \delta_{2} \Gamma^{\prime} \cup \cdots \cup \delta_{k} \Gamma^{\prime}$, where $\delta_{i} \Gamma^{\prime}$ are distinct cosets, as $\Gamma$ is finite. We will find a bound on $k$. Note that if $\left\|\delta_{i}-\delta_{j}\right\|<\frac{1}{2}$, then $\left\|\delta_{j}^{-1} \delta_{i}-\mathrm{Id}\right\|=\left\|\delta_{j}^{-1}\left(\delta_{i}-\delta_{j}\right)\right\|=\left\|\delta_{i}-\delta_{j}\right\|<\frac{1}{2}$, since $\delta_{j}^{-1} \in O(n+1)$. Hence, $\delta_{j}^{-1} \delta_{i} \in U \cap \Gamma^{\prime}$. Therefore, $\delta_{i}=\delta_{j}\left(\delta_{j}^{-1} \delta_{i}\right) \in \delta_{j} \Gamma^{\prime}$, and so $\delta_{i}$ and $\delta_{j}$ are in the same coset. Since we have written $\Gamma$ as a union of distinct cosets, then the $\delta_{i}$ s are all at least $\frac{1}{2}$ away from each other in $O(n+1)$. Place disjoint $\frac{1}{2}$ balls in $S O(n+1)$ about each $\delta_{i}$. Now $k$, the index of $\Gamma^{\prime}$ in $\Gamma$, must be less than or equal to the maximum number of disjoint $\frac{1}{2}$ balls we can put in $S O(n+1)$. Using volume estimates, it follows that $k$ is bounded above by a constant depending only on the dimension $n$, call it $k$.

Finally, we will find a lower bound for the diameter of $S^{n} / \Gamma$. Since $\Gamma^{\prime}$ is abelian, we know that the diameter of $S^{n} / \Gamma^{\prime}$ is $\pi / 2$ or $\pi$, by Lemma 3.6. Let $d$ be the diameter of $S^{n} / \Gamma$. From Lemma 3.13 we know that $d \geq \pi / 4(k-1)$, where $k$ depends only on $n$, as desired.

Corollary 3.14.1. If $\Gamma$ is a finite subgroup of the isometry group of $M^{n}$, where $M^{n}$ is $\mathbb{C} P^{n}$ or $\mathbb{U} P^{n}$, then there exists $\varepsilon$ depending only on $n$, so that $\operatorname{diam}\left(M^{n} / \Gamma\right) \geq$ $\varepsilon(n)$.

Proof. Fix $n$. The proof is an extension of Theorem 3.14 via a Hopf-fibration argument.

For $\mathbb{C} P^{n}$, let $\Gamma_{i}^{c} \subset \operatorname{Isom}\left(\mathbb{C} P^{n}\right)=S U(n+1) / \mathbb{Z}_{n+1} \cup c S U(n+1) / \mathbb{Z}_{n+1}$, where $c$ is complex conjugation, be finite. Recall that $\mathbb{C} P^{n}$ is isometric to $S^{2 n+1} / U(1)$ via the submersion metric. One obtains the Hopf-fibration from the diagonal embedding of $U(1)$ into $S U(n+1)$. Now, look at the inverse image of $\Gamma_{i}^{c}$ in $O(2 n+2)$ via lifting to $S U(n+1) \cup c S U(n+1)$, and call it $\Gamma_{i}$. Find a finite abelian subgroup of $\Gamma_{i}$ as in in Theorem 3.14, and call it $\Gamma_{i}^{\prime}$. We know that the index, $k$, of $\Gamma_{i}^{\prime}$ in $\Gamma_{i}$ depends only on $n$. Diagonalize the $\Gamma_{i}$ matrices over $\mathbb{C}$. We will now apply $U(1)$ from the Hopf fibration. Notice that $\Gamma_{i}^{\prime} \cdot U(1)$ will also have 
index $k$ in $\Gamma_{i} \cdot U(1)$. Since $\Gamma_{i}$ and $U(1)$ are all diagonal, they preserve an axis. Hence, they are reducible. Then, $\operatorname{diam}\left(S^{2 n+1} / \Gamma_{i}^{\prime} \cdot U(1)\right)$ is at least $\pi / 2$. We use the index $k$, depending only on $n$, to obtain a lower bound on the diameter of $S^{2 n+1} / \Gamma_{i} \cdot U(1)=\left(S^{2 n+1} / U(1)\right) / \Gamma_{i}=\mathbb{C} P^{n} /\left(\Gamma_{i} / \mathbb{Z}_{n+1}\right)$, as desired.

For $\mathbb{H} P^{n}$, let $\Gamma_{i}^{h} \subset \operatorname{Isom}\left(\mathbb{H} P^{n}\right)=S p(n+1) / \mathbb{Z}_{2}$ be finite. The Hopf fibration $S^{4 n+3} \rightarrow \mathbb{H} P^{n}$ embeds $S p(1)$ into $S p(n+1) \subset S O(4 n+4)$. The argument follows by pulling $\Gamma_{i}^{h}$ back into $S O(4 n+4)$ and continuing as above. Once diagonalizing $\Gamma_{i}^{\prime}$, we see that $\Gamma_{i}^{\prime}$ and $S p(1)$ preserve an axis, since $\Gamma_{i}^{\prime}$ acts on the left and $S p(1)$ acts on the right. Hence, the action is reducible and the argument follows as above.

3.4. Coxeter groups. In this section, we will prove that if $\Gamma$ is a Coxeter group, a group generated by reflections (see [24] and [22] for background information), then $\operatorname{diam}\left(S^{n} / \Gamma\right) \geq \pi / 8.10$.

The Weyl group of a Lie group is a Coxeter group, so Coxeter groups are of natural interest. To examine the meaning of the diameter lower bound for Lie groups, let $G$ be a compact Lie group of dimension $n+1$. Look at a compact torus $T$ in $G$. On the Lie algebra level, $\mathfrak{t} \subset \mathfrak{g}$, as a maximal abelian subalgebra. Let $N(T)$ be the normalizer of $T$. Now, the Weyl group $W=N(T) / T$ acts on $\mathfrak{t}$ via conjugation. Let $\operatorname{Ad}(G)$ be the adjoint action of $G$ on $\mathfrak{g}$. There exists a bi-invariant metric on the Lie algebra so that $\operatorname{Ad}(G)$ acts by isometries. Examine $S^{n}(1) \subset \mathfrak{g}$, where $S^{n}(1)$ has radius one. Now, $S^{n}(1) / \operatorname{Ad}(G)=\left(S^{n}(1) \cap \mathfrak{t}\right) / W$. Hence, a lower bound on the diameter of $\left(S^{n}(1) \cap \mathfrak{t}\right) / W$ gives a lower bound on the diameter of $S^{n}(1) / \operatorname{Ad}(G)$.

Notation and background. Let $r$ be a non-trivial vector in $\mathbb{R}^{n+1}$ and define $H_{r}$ as the subspace orthogonal to $r$. A reflection $R_{r}$ in $\mathbb{R}^{n+1}$ sends $r$ to $-r$, fixes $H_{r}$, and sends any vector $v$ to

$$
v-\frac{\langle v, r\rangle}{\langle r, r\rangle} r
$$

Notice that $R_{r} \in O(n+1)$. A Coxeter group $\Gamma$ is a finite group generated by reflections.

A root system $R$ for $\Gamma$ is a finite set of nonzero vectors in $\mathbb{R}^{n+1}$ so that each vector $r$, called a root, satisfies:

$$
\begin{gathered}
\forall c \in \mathbb{R}, \mathrm{R} \cap c r=\{r,-r\}, \\
R_{r}(\mathrm{R})=\mathrm{R} .
\end{gathered}
$$

A simple system $\Delta$, with simple roots $r_{i}$, is a real basis for the root system so that each root vector in $\mathrm{R}$ is a linear combination of simple roots, where the coefficients all have the same sign. Every Coxeter group has a simple system [24], so let 


$$
\Delta_{\Gamma}=\left\{r_{1}, r_{2}, \ldots, r_{m}\right\},
$$

where $r_{i}$ is a simple root of the Coxeter group $\Gamma$. $\Gamma$ is generated by the reflections $R_{r_{i}}$ [22]. Let

$$
D=\left\{v \in \mathbb{R}^{n+1} \mid\left\langle v, r_{i}\right\rangle \geq 0 \text { for all simple } r_{i}\right\} .
$$

$D$ is a fundamental domain for $\Gamma$ and there are no further identifications on the boundary of $D$ [24, pp. 22-23]. A dual basis $q_{i}$ for $r_{i}$, where $\left\langle q_{i}, r_{j}\right\rangle=\delta_{i j}$, forms the vertices of the fundamental domain [22]. Hence, $D \cap S^{n}$ is a fundamental domain for the action of $\Gamma$ on $S^{n}$.

All irreducible Coxeter groups, groups for which $\Delta_{\Gamma}$ is not the union of two nonempty orthogonal subsets, are classified in [22] via the Coxeter diagram and its corresponding simple roots ([22, p. 71]). These are $H_{n}^{2}, G_{2}, I_{3}, I_{4}, F_{4}, E_{6}, E_{7}$, $E_{8}, A_{n}, B_{n}$, and $D_{n}$. We use the simple roots $r_{i}$ to compute a dual basis $q_{i}$ as follows. Let $A$ be the matrix with $\left\langle r_{i}, r_{j}\right\rangle$ as the $i j$ th entry, and let $A_{(i j)}^{-1}$ be the $i j$ th entry of $A^{-1}$. Then

$$
q_{i}=\sum_{j=1}^{3} A_{(i j)}^{-1} r_{j}
$$

is a dual basis vector for $\Gamma\left[22\right.$, p. 52]. Since the dual basis vectors $q_{i}$ are the vertices of the fundamental domain, and the fundamental domain has no additional identifications on it, the diameter of $S^{n} / \Gamma$ is achieved as the largest spherical distance between dual basis vectors. Since spherical distance is the angle, we look for the two basis vectors with maximum angle between them. After a description of each group and the dual basis vectors, we give the two vectors that achieve the diameter, and also give the value for the diameter. Notice that we eliminate the dihedral groups $H_{n}^{2}$ and $G_{2}$ from the list because, as Coxeter groups, they act on $\mathbb{R}^{2}$.

Define $\alpha=2 \cos (\pi / 5)$ and $\beta=\cos (2 \pi / 5)$.

I3. $\quad I_{3}$ has order $120=2^{3} 3 \cdot 5$. It acts on $S^{2}$ as $I^{-}$, the Coxeter extension of $I$, the icosahedral group. The simple root vectors are

$$
\begin{aligned}
& r_{1}=[\beta \alpha+\beta, \beta,-\beta \alpha], \\
& r_{2}=[-\beta \alpha-\beta, \beta, \beta \alpha], \\
& r_{3}=[\beta \alpha,-\beta \alpha-\beta, \beta] .
\end{aligned}
$$

The dual basis vectors are 


$$
\begin{aligned}
& q_{1}=\left[-\frac{\sqrt{5}-1}{-3+\sqrt{5}},-\frac{\sqrt{5}-1}{-3+\sqrt{5}},-\frac{\sqrt{5}-1}{-3+\sqrt{5}}\right], \\
& q_{2}=\left[1,-\frac{\sqrt{5}-1}{-3+\sqrt{5}},-\frac{2}{-3+\sqrt{5}}\right], \\
& q_{3}=\left[1,0,-\frac{\sqrt{5}-1}{-3+\sqrt{5}}\right] .
\end{aligned}
$$

The diameter of $S^{2} / I_{3}$ is achieved by $q_{1}$ and $q_{3}$ and is $\arccos \left(\frac{1}{3} \sqrt{3} / \sqrt{5-2 \sqrt{5}}\right)=$ $\arccos [\tan (3 \pi / 10) / \sqrt{3}] \approx \pi / 4.82$.

I4. $I_{4}$ has order $120^{2}=2^{6} 3^{2} 5^{2}$. It acts on $S^{3}$ as $\left(I^{*} \times I^{*}\right)^{-}$, the Coxeter extension of $I^{*} \times I^{*}$ (see [14, p. 57]), where $I^{*}$ is as in "Manifolds and the diameter" on page 1455 . The simple root vectors are

$$
\begin{array}{ll}
r_{1}=[\beta \alpha+\beta, \beta,-\beta \alpha, 0], & r_{2}=[-\beta \alpha-\beta, \beta, \beta \alpha, 0], \\
r_{3}=[\beta \alpha,-\beta \alpha-\beta, \beta, 0], & r_{4}=[-\beta \alpha, 0,-\beta \alpha-\beta, \beta] .
\end{array}
$$

The dual basis vectors are

$$
\begin{aligned}
& q_{1}=\left[-\frac{\sqrt{5}-1}{-3+\sqrt{5}},-\frac{\sqrt{5}-1}{-3+\sqrt{5}},-\frac{\sqrt{5}-1}{-3+\sqrt{5}},-\frac{2}{-7+3 \sqrt{5}}\right], \\
& q_{2}=\left[1,-\frac{\sqrt{5}-1}{-3+\sqrt{5}},-\frac{2}{-3+\sqrt{5}},-\frac{2 \sqrt{5}-2}{-7+3 \sqrt{5}}\right], \\
& q_{3}=\left[1,0,-\frac{\sqrt{5}-1}{-3+\sqrt{5}},-\frac{3 \sqrt{5}-5}{-7+3 \sqrt{5}}\right], \\
& q_{4}=\left[0,0,0, \frac{-4 \sqrt{5}-2}{-7+3 \sqrt{5}}\right] .
\end{aligned}
$$

The diameter of $S^{3} / I_{4}$ is achieved by $q_{1}$ and $q_{4}$ and is

$$
\pi-\arccos \left[\frac{\sqrt{2}(-9+4 \sqrt{5})}{(-7+3 \sqrt{5})^{2}}\right] \approx \frac{\pi}{8.10} .
$$

$\underline{F_{4}} . \quad F_{4}$ has order $2^{7} 3^{2}$. It acts on $S^{3}$ as follows. This is the group of symmetries of a regular solid in $\mathbb{R}^{4}$ having 24 (three-dimensional) faces which are octahedra [11]. The simple root vectors are

$$
\begin{array}{ll}
r_{1}=\left[\frac{-1}{2}, \frac{-1}{2}, \frac{-1}{2}, \frac{-1}{2}\right], & r_{2}=[1,0,0,0], \\
r_{3}=[-1,1,0,0], & r_{4}=[0,-1,1,0] .
\end{array}
$$


The dual basis vectors are

$$
\begin{array}{ll}
q_{1}=[0,0,0,-2], & q_{2}=[1,1,1,-3], \\
q_{3}=[0,1,1,-2], & q_{4}=[0,0,1,-1] .
\end{array}
$$

The diameter of $S^{3} / F_{4}$ is achieved by $q_{1}$ and $q_{4}$ and is $\pi / 4$.

$E_{6} . \quad E_{6}$ has order $2^{7} 3^{4} 5$ and acts on $S^{5}$. It is the group of automorphisms of a configuration of 27 lines on a cubic surface. The simple root vectors are

$$
\begin{array}{ll}
r_{1}=\left[\frac{1}{2}, \frac{1}{2}, \frac{1}{2}, \frac{-1}{2}, \frac{-1}{2}, \frac{-1}{2}, \frac{-1}{2}, \frac{-1}{2}\right], & r_{2}=[-1,1,0,0,0,0,0,0], \\
r_{3}=[0,-1,1,0,0,0,0,0], & r_{4}=[0,0,-1,1,0,0,0,0], \\
r_{5}=[0,0,0,-1,1,0,0,0], & r_{6}=[0,0,0,0,-1,1,0,0] .
\end{array}
$$

The dual basis vectors are

$$
\begin{aligned}
& q_{1}=[0,0,0,0,0,0,-1,-1], \\
& q_{2}=\left[\frac{-5}{6}, \frac{1}{6}, \frac{1}{6}, \frac{1}{6}, \frac{1}{6}, \frac{1}{6}, \frac{-1}{2}, \frac{-1}{2}\right], \\
& q_{3}=\left[\frac{-2}{3}, \frac{-2}{3}, \frac{1}{3}, \frac{1}{3}, \frac{1}{3}, \frac{1}{3},-1,-1\right], \\
& q_{4}=\left[\frac{-1}{2}, \frac{-1}{2}, \frac{-1}{2}, \frac{1}{2}, \frac{1}{2}, \frac{1}{2}, \frac{-3}{2}, \frac{-3}{2}\right], \\
& q_{5}=\left[\frac{-1}{3}, \frac{-1}{3}, \frac{-1}{3}, \frac{-1}{3}, \frac{2}{3}, \frac{2}{3},-1,-1\right], \\
& q_{6}=\left[\frac{-1}{6}, \frac{-1}{6}, \frac{-1}{6}, \frac{-1}{6}, \frac{-1}{6}, \frac{5}{6}, \frac{-1}{2}, \frac{-1}{2}\right] .
\end{aligned}
$$

The diameter of $S^{5} / E_{6}$ is achieved by $q_{2}$ and $q_{6}$ and is $\pi / 3$.

$\underline{E_{7}} . E_{7}$ has order $2^{10} 3^{4} 5 \cdot 7$ and acts on $S^{6}$. The simple root vectors are

$$
\begin{array}{ll}
r_{1}=\left[\frac{1}{2}, \frac{1}{2}, \frac{1}{2}, \frac{-1}{2}, \frac{-1}{2}, \frac{-1}{2}, \frac{-1}{2}, \frac{-1}{2}\right], & r_{2}=[-1,1,0,0,0,0,0,0], \\
r_{3}=[0,-1,1,0,0,0,0,0], & r_{4}=[0,0,-1,1,0,0,0,0], \\
r_{5}=[0,0,0,-1,1,0,0,0], & r_{6}=[0,0,0,0,-1,1,0,0], \\
r_{7}=[0,0,0,0,0,-1,1,0] . &
\end{array}
$$


The dual basis vectors are

$$
\begin{aligned}
& q_{1}=\left[\frac{-1}{4}, \frac{-1}{4}, \frac{-1}{4}, \frac{-1}{4}, \frac{-1}{4}, \frac{-1}{4}, \frac{-1}{4}, \frac{-7}{4}\right], \\
& q_{2}=[-1,0,0,0,0,0,0,-1], \\
& q_{3}=[-1,-1,0,0,0,0,0,-2], \\
& q_{4}=[-1,-1,0,0,0,0,0,-3], \\
& q_{5}=\left[\frac{-3}{4}, \frac{-3}{4}, \frac{-3}{4}, \frac{-3}{4}, \frac{1}{4}, \frac{1}{4}, \frac{1}{4}, \frac{-9}{4}\right], \\
& q_{6}=\left[\frac{-1}{2}, \frac{-1}{2}, \frac{-1}{2}, \frac{-1}{2}, \frac{-1}{2}, \frac{1}{2}, \frac{1}{2}, \frac{-3}{2}\right], \\
& q_{7}=\left[\frac{-1}{4}, \frac{-1}{4}, \frac{-1}{4}, \frac{-1}{4}, \frac{-1}{4}, \frac{-1}{4}, \frac{3}{4}, \frac{-3}{4}\right] .
\end{aligned}
$$

The diameter of $S^{6} / E_{7}$ is achieved by $q_{2}$ and $q_{7}$ and is $\arccos (\sqrt{3} / 3) \approx \pi / 3.29$.

$\underline{E_{8}} . E_{8}$ has dimension $2^{14} 3^{5} 5^{2} 7$ and acts on $S^{7}$. The simple root vectors are

$$
\begin{array}{ll}
r_{1}=\left[\frac{1}{2}, \frac{1}{2}, \frac{1}{2}, \frac{-1}{2}, \frac{-1}{2}, \frac{-1}{2}, \frac{-1}{2}, \frac{-1}{2}\right], & r_{2}=[-1,1,0,0,0,0,0,0], \\
r_{3}=[0,-1,1,0,0,0,0,0], & r_{4}=[0,0,-1,1,0,0,0,0], \\
r_{5}=[0,0,0,-1,1,0,0,0], & r_{6}=[0,0,0,0,-1,1,0,0], \\
r_{7}=[0,0,0,0,0,-1,1,0], & r_{8}=[0,0,0,0,0,0,-1,1] .
\end{array}
$$

The dual basis vectors are

$$
\begin{aligned}
& q_{1}=[-1,-1,-1,-1,-1,-1,-1,-1], \\
& q_{2}=\left[\frac{-3}{2}, \frac{-1}{2}, \frac{-1}{2}, \frac{-1}{2}, \frac{-1}{2}, \frac{-1}{2}, \frac{-1}{2}, \frac{-1}{2}\right], \\
& q_{3}=[-2,-2,-1,-1,-1,-1,-1,-1], \\
& q_{4}=\left[\frac{-5}{2}, \frac{-5}{2}, \frac{-5}{2}, \frac{-3}{2}, \frac{-3}{2}, \frac{-3}{2}, \frac{-3}{2}, \frac{-3}{2}\right], \\
& q_{5}=[-2,-2,-2,-2,-1,-1,-1,-1], \\
& q_{6}=\left[\frac{-3}{2}, \frac{-3}{2}, \frac{-3}{2}, \frac{-3}{2}, \frac{-3}{2}, \frac{-1}{2}, \frac{-1}{2}, \frac{-1}{2}\right], \\
& q_{7}=[-1,-1,-1,-1,-1,-1,0,0], \\
& q_{8}=\left[\frac{-1}{2}, \frac{-1}{2}, \frac{-1}{2}, \frac{-1}{2}, \frac{-1}{2}, \frac{-1}{2}, \frac{-1}{2}, \frac{1}{2}\right] .
\end{aligned}
$$

The diameter of $S^{7} / E_{8}$ is achieved by $q_{2}$ and $q_{8}$ and is $\pi / 4$.

$\underline{A_{n}} . A_{n}$ has order $(n+1)$ ! and acts on $S^{n-1}$ as follows. Consider the symmetric group $S_{n+1}$ acting as permutations on the coordinates of $\mathbb{R}^{n+1}$. Notice that $S_{n+1}$ fixes the line corresponding to $e_{1}+e_{2}+\cdots+e_{n+1}$, where $e_{i}$ is the standard basis vector in $\mathbb{R}^{n+1}$. The orthogonal plane consisting of vectors whose coordinates add up to 0 is left invariant under $S_{n+1}$. Let $A_{n}$ be the action of $S_{n+1}$ restricted to this orthogonal plane. $A_{n}$ fixes the origin in this new $\mathbb{R}^{n}$, and so it 
acts on $S^{n-1}$. Notice that $A_{n}$ is not the group of even permutations. The simple root vectors are

$$
\begin{gathered}
r_{1}=[-1,1,0, \ldots, 0,0,0], \\
r_{2}=[0,-1,1,0, \ldots, 0,0], \\
r_{3}=[0,0,-1,1,0, \ldots, 0], \\
\vdots \\
r_{n}=[0,0,0,0, \ldots,-1,1] .
\end{gathered}
$$

The dual basis vectors are

$$
\begin{aligned}
q_{1} & =\left[\frac{-n}{n+1}, \frac{1}{n+1}, \frac{1}{n+1}, \ldots, \frac{1}{n+1}\right], \\
q_{2} & =\left[\frac{-(n-1)}{n+1}, \frac{-(n-1)}{n+1}, \frac{2}{n+1}, \ldots, \frac{2}{n+1}\right], \\
q_{3} & =\left[\frac{-(n-2)}{n+1}, \frac{(n-2)}{n+1}, \frac{(n-2)}{n+1}, \frac{3}{n+1}, \ldots, \frac{3}{n+1}\right], \\
q_{n-1} & =\left[\frac{-2}{n+1}, \frac{-2}{n+1}, \ldots, \frac{-2}{n+1}, \frac{n-1}{n+1}, \frac{n-1}{n+1}\right], \\
q_{n} & =\left[\frac{-1}{n+1}, \frac{-1}{n+1}, \ldots, \frac{-1}{n+1}, \frac{n}{n+1}\right] .
\end{aligned}
$$

The diameter of $S^{n-1} / A_{n}$ is achieved by $q_{1}$ and $q_{n}$ and is $\arccos (1 / n)$, which goes to $\pi / 2$ as $n \rightarrow \infty$. The diameter of $S^{2} / A_{3}$ is $\arccos \frac{1}{3} \approx \pi / 2.56 . A_{3}$ is the group $T^{-}$, the full isometry group of a regular tetrahedron including reflection symmetries, as in [20, pp. 18-20].

$\underline{B_{n}} . B_{n}$ has order $2^{n} n$ ! and acts on $S^{n-1}$ as follows. Let $S_{n}$ be as in the description of $A_{n}$ above. The sign change reflections sending $e_{i}$ to its negative and fixing all other $e_{j}$ generate a group of order $2^{n}$ isomorphic to $\mathbb{Z}_{2}^{n}$. Conjugating a sign change by a transposition will yield another sign change. $B_{n}$ is the semi-direct product of $S_{n}$ and $\mathbb{Z}_{2}^{n}$. The simple root vectors are

$$
\begin{gathered}
r_{1}=[1,0,0, \ldots, 0,0,0], \\
r_{2}=[-1,1,0, \ldots, 0,0,0], \\
r_{3}=[0,-1,1,0, \ldots, 0,0], \\
r_{4}=[0,0,-1,1,0, \ldots, 0], \\
\quad \vdots \\
r_{n}=[0,0,0,0, \ldots,-1,1] .
\end{gathered}
$$


The dual basis vectors are

$$
\begin{gathered}
q_{1}=[1,1,1, \ldots, 1,1,1], \\
q_{2}=[0,1,1, \ldots, 1,1,1], \\
q_{3}=[0,0,1, \ldots, 1,1,1], \\
\vdots \\
q_{n}=[0,0,0, \ldots, 0,0,1] .
\end{gathered}
$$

The diameter of $S^{n-1} / B_{n}$ is achieved by $q_{1}$ and $q_{n}$ and is $\arccos (\sqrt{n} / n)$. As $n \rightarrow \infty$, the diameter approaches $\pi / 2$. The diameter of $S^{2} / B_{3}$, is $\arccos (\sqrt{3} / 3) \approx$ $\pi / 3.29 . B_{3}$ is the group $O^{-}$, the orthogonal extension of the octahedral group [20, pp. 20-21].

$D_{n} . D_{n}$ has order $2^{n-1} n !$ and acts on $S^{n-1} . D_{n}$ is a subgroup of index 2 in $B_{n}$. Look at the subgroup of $\mathbb{Z}_{2}^{n}$ consisting only of sign changes that involve an even number of signs, which is generated by $e_{i}+e_{j} \rightarrow-\left(e_{i}+e_{j}\right)$ for $i \neq j . D_{n}$ is the semi-direct product of this subgroup with $S_{n}$. The simple root vectors are

$$
\begin{gathered}
r_{1}=[1,1,0, \ldots, 0,0,0], \\
r_{2}=[-1,1,0, \ldots, 0,0,0], \\
r_{3}=[0,-1,1,0, \ldots, 0,0], \\
\vdots \\
r_{n}=[0,0,0,0, \ldots,-1,1] .
\end{gathered}
$$

The dual basis vectors are

$$
\begin{aligned}
& q_{1}=\left[\frac{1}{2}, \frac{1}{2}, \frac{1}{2}, \ldots, \frac{1}{2}, \frac{1}{2}, \frac{1}{2}\right], \\
& q_{2}=\left[\frac{-1}{2}, \frac{1}{2}, \frac{1}{2}, \ldots, \frac{1}{2}, \frac{1}{2}, \frac{1}{2}\right], \\
& q_{3}=[0,0,1,1, \ldots, 1,1], \\
& q_{4}=[0,0,0,1, \ldots, 1,1], \\
& \vdots \\
& q_{n}=[0,0,0,0, \ldots, 0,1] .
\end{aligned}
$$

For $n \geq 4$, the diameter of $S^{n-1} / D_{n}$ is achieved by $q_{1}$ and $q_{n}$ and is $\arccos (\sqrt{n} / n)$. As $n \rightarrow \infty$, the diameter approaches $\pi / 2$. For $n=3$, the diameter of $S^{2} / D_{3}$ is achieved by $q_{1}$ and $q_{2}$ and is $\arccos \frac{1}{3} \approx \pi / 2.56$. Notice that $D_{3}\left(=A_{3}\right)$ is the group $T^{-}$as described above. 


\begin{tabular}{|c|c|c|}
\hline \multicolumn{3}{|c|}{ SuMMARY OF COXETER ORBIFOLd DiAMETER RESUltS } \\
\hline $\begin{array}{l}\text { Coxeter } \\
\text { Group }\end{array}$ & $\begin{array}{c}\text { Other Group Descriptions } \\
\text { (compare with [14, pp. 57-61], } \\
[16,12], \text { and }[22, \mathrm{pp} .81])\end{array}$ & $\begin{array}{l}\text { Resulting } \\
\text { Diameter Lower } \\
\text { Bound }\end{array}$ \\
\hline$I_{3}$ & Icosahedral $I^{-}$ & $\pi / 4.82$ \\
\hline$I_{4}$ & $\left(I^{*} \times I^{*}\right)^{-}$ & $\pi / 8.10$ \\
\hline$F_{4}$ & Index-2 extension of $O^{*} \times{ }_{C_{2}} O^{*}$ & $\pi / 4$ \\
\hline$E_{6}$ & Automorphisms of 27 lines on a cubic $\mathbb{P}_{6}^{2}$ & $\pi / 3$ \\
\hline$E_{7}$ & Automorphisms of 56 lines on $\mathbb{P}_{7}^{2}$ & $\pi / 3.29$ \\
\hline$E_{8}$ & Automorphisms of 240 lines on $\mathbb{P}_{8}^{2}$ & $\pi / 4$ \\
\hline$A_{n}$ & & $\arccos (1 / n)$ \\
\hline$B_{n}$ & 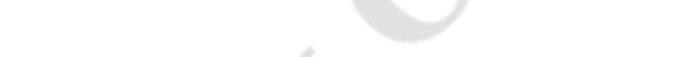 & $\arccos (\sqrt{n} / n)$ \\
\hline$D_{n}, n>3$ & 201. & $\arccos (\sqrt{n} / n)$ \\
\hline$A_{3}=D_{3}$ & Tetrahedral $T^{-}$ & $\pi / 2.56$ \\
\hline$B_{3}$ & Octahedral $O^{-}$ & $\pi / 3.29$ \\
\hline$A_{4}$ & Index-2 extension of $I^{*} \times_{\check{I}} I^{* 1}$ & $\pi / 2.38$ \\
\hline$B_{4}$ & $\left(O^{*} \times_{D_{3}} O^{*}\right)^{-}$ & $\pi / 3$ \\
\hline$D_{4}$ & Index- 2 extension of $T^{*} \times_{C_{3}} T^{*}$ & $\pi / 3$ \\
\hline
\end{tabular}

TABLE 1. Summary of Coxeter orbifold diameter results

\section{Lower bound for Coxeter groups.}

Theorem 3.15. If $\Gamma$ is a Coxeter group, generated by reflections, then $\operatorname{diam}\left(S^{n} / \Gamma\right) \geq \pi / 8.10$.

Proof. If $\Gamma$ is a reducible Coxeter group, then $\Delta_{\Gamma}$ is the union of two nonempty orthogonal subsets $A$ and $B$ by definition. Look at the subspaces $H_{A}$ and $H_{B}$ generated by $A$ and $B$, respectively. We claim these subspaces are invariant under $\Gamma$. Notice that for any $b \in B, R_{b}$ fixes $H_{A}$ by definition of $R_{b}$, since $H_{A} \subset H_{b}$. Now $H_{A}^{\perp}=H_{b}$, and $\Gamma$ acts by isometries. Hence, $H_{b}$ leaves $H_{B}$ invariant also. Similarly, $H_{a}$ leaves both $H_{A}$ and $H_{B}$ invariant. Since $\Gamma$ is reducible in the sense of invariant subspaces, we know that the diameter of $S^{n} / \Gamma \geq \pi / 2$.

For every irreducible Coxeter group, we have found a dual basis and then exhaustively computed the diameter of $S^{n} / \Gamma$. Notice that the lowest diameter 
occurs in dimension three. This is achieved as the diameter of the quotient of $S^{3}$ by $I_{4}$, the Coxeter group extension of $I^{*} \times I^{*}$. Given a fixed dimension $n$ which is large, $A_{n+1}, B_{n+1}$, and $D_{n+1}$ are the only Coxeter groups which act on $S^{n}$. Since $A_{n+1}$ has a resulting diameter of $\arccos (1 /(n+1))$, while $B_{n+1}$ and $D_{n+1}$ each have resulting diameter $\arccos (\sqrt{n+1} /(n+1))$, we see that the smallest diameter is achieved by both $B_{n+1}$ and $D_{n+1}$. This smallest diameter, $\arccos (\sqrt{n+1} /(n+1))$, increases monotonically in $n$. As $n$ approaches infinity, the diameter approaches $\pi / 2$.

\section{INFINITE GROUPS}

\subsection{Intuition and examples.}

Cohomogeneity-one actions and their resulting diameter. When the quotient space is an interval, the action is called a cohomogeneity-one action. In Example 3.1, we saw that the quotient of $S^{2}$ by $S^{1}$, the maximal torus of $S O$ (3), is a longitude of length $\pi$. In general, since the orbits $G p$ are isoparametric hypersurfaces in spheres, it is well known that the length of the intervals are $\pi / p$ where $p=2,3,4$, or 6 ([23]). Hence, the diameter is at least $\pi / 6$. There are only two examples, in dimensions 7 and 13, where the diameter is equal to $\pi / 6$. However, unlike the manifold and Coxeter orbifold cases, there is an entire class of cohomogeneity-one actions on $S^{n}$, including actions for arbitrarily large dimensions, which result in a quotient space of diameter $\pi / 4$.

Arbitrary actions. If the group is infinite, then the resulting quotient space is an Alexandrov space with curvature bounded below. The explicit orbifold lower bounds in Theorem 1 do not apply since discreteness was needed in the proof.

Example 4.1. $\mathbb{C} P^{n}$. Look at the Hopf action on $S^{2 n+1}$. Then $S^{2 n+1} / S^{1}=$ $\mathbb{C} P^{n}$ has diameter $\pi / 2$.

Example 4.2. $S^{3} / S^{1} \times I^{*}$. As in "Manifolds and the diameter" on page 1455, $S^{1} \times I^{*}$ acts on $S^{3}$ by quaternionic left and right multiplication. Now, $\operatorname{diam}\left(S^{3} / S^{1} \times I^{*}\right)=\operatorname{diam}\left(\mathbb{C} P^{1} / I\right)=\operatorname{diam}\left(S^{2}\left(\frac{1}{2}\right) / I\right)$, where $S^{2}(1 / 2)$ is the 2sphere of radius $\frac{1}{2}$.

Since $\operatorname{diam}\left(S^{2} / I\right)=\arccos (\tan (3 \pi / 10) / \sqrt{3})$, which is approximately $\pi / 4.82$, we see that $\operatorname{diam}\left(S^{2}\left(\frac{1}{2}\right) / I\right)=.5 \arccos (\tan (3 \pi / 10) / \sqrt{3})$, which is approximately $\pi / 9.63$.

4.2. Infinite group conditions. Let $G$ be infinite. If $G$ is transitive, then $S^{n} / G$ is a point, which has diameter 0 . Hence, we restrict to non-transitive actions.

The diameter resulting from a group is smaller than or equal to the diameter resulting from its subgroups, so for any subgroup, we restrict to its closure. This restriction is important, since if $G$ is not closed, then the quotient is not even Hausdorff. For example, look at the group $G \subset S O(3)$ acting on $S^{2}$, generated 
as follows. Let $r_{9}$ be a $2 \times 2$ real rotation matrix with rotation angle an irrational multiple of $\pi$. Let $G$ be generated by $\left(\begin{array}{cc}r_{9} & 0 \\ 0 & 1\end{array}\right)$ and $\left(\begin{array}{ll}1 & 0 \\ 0 & r_{9}\end{array}\right)$. This action is not transitive on $S^{2}$, but any point gets arbitrarily close to any other point.

In general, given generators, it can be quite difficult to decide if the resulting group is finite or infinite. For example, for the two generators above, let $\vartheta$ be a rational multiple of $\pi$. Notice that $G$ is not cyclic or dihedral. The largest order of any irreducible, finite group in $O(3)$ acting on $S^{2}$ is $60=|I|$ [1]. Hence, if $\vartheta$ is small enough, then $G$ is infinite.

4.3. Existence of a lower bound in a fixed dimension. In this section we will prove the following theorem.

Theorem 4.3. If $G$ is a non-transitive group, then there exists $\varepsilon$, depending only on $n$, so that $\operatorname{diam}\left(S^{n} / G\right) \geq \varepsilon(n)$.

The lower bounds are not explicit since the proof is by contradiction.

In the proof, we show that a non-transitive sequence of groups cannot converge to a transitive subgroup of $S O(n+1)$. The following example is good to keep in mind while reading the details of the proof, since we must show that this behavior cannot occur on the sphere (compare with Lemma 4.9).

Example 4.4. Let $T^{2}$ be a torus. Let $G_{i}$ be lines of rational slope converging (in the sense of Lemma 4.5) to a line of irrational slope.

Now, $G_{i}$ are non-transitive groups converging to a transitive group on $T^{2}$. Note that the corresponding Lie algebras are all dimension 1, and as subspaces, they converge to a dimension 1 space. The Lie algebras are all abelian.

Lemma 4.5. Let $G$ be a compact Lie group, and let $G_{i}$ be a sequence of closed subgroups of $G$. Then

(1) There exists a subsequence and a compact group $G_{\infty}$ such that

(a) $G_{\infty}=$ \{limit of any convergent sequence $g_{i}$, where $g_{i} \in G_{i}$ for all $i$. $\}$

(b) $G_{\infty}$ does not get any larger when a smaller subsequence is taken.

(c) for every neighborhood $U$ of $G_{\infty}$, there exists $i_{o}$ so that $G_{i} \subset U$ for $i \geq i_{o}$.

(2) There exists a further subsequence so that $G_{i}$ is conjugate to $G_{i}^{\prime} \subset G_{\infty}$ in $G$, where $G_{i}^{\prime}$ converges to $G_{\infty}$ in the sense of $(1 \mathrm{a})$.

Notation. We say that $G_{i} \rightarrow G_{\infty}$ if $G_{i}$ converges to $G_{\infty}$ in the sense of (1). We say that $G_{i} \hookrightarrow G_{\infty}$ if $G_{i} \rightarrow G_{\infty}$ and $G_{i} \subset G_{\infty}$ as in (2).

In general, for a Lie group $H$, let $H^{o}$ be the connected component of the identity of $H$. Denote $Z(H)$ as the center of $H$, and $\mathfrak{Z}(\mathfrak{h})$ as the center of the corresponding Lie algebra $\mathfrak{h}$. Let $\mathfrak{h}^{s \mathcal{S}}$ be the semi-simple part of $\mathfrak{h}$. Every Lie algebra of a compact Lie group can be decomposed into its center and semi-simple ideals. Thus, $\mathfrak{h}=\mathfrak{h}^{s \mathcal{S}}+\mathfrak{Z}(\mathfrak{h})$. Denote $H^{s s}=\exp \left(\mathfrak{h}^{s \mathcal{S}}\right)$ as the semi-simple part of $H^{o}$. 
Proof of (1). Let $G_{i}$ be a sequence of closed subgroups of a compact group $G$. Define $G_{\infty}$ as in (1a).

To show that $G_{\infty}$ is a group, first notice that multiplication and $g \rightarrow g^{-1}$ are continuous in $G$. So, given $g_{\infty}^{1}, g_{\infty}^{2} \in G_{\infty}$, define $g_{\infty}^{1} g_{\infty}^{2}$ by the limit of the sequence $g_{i}^{1} g_{i}^{2}$, where $g_{i}^{1} \rightarrow g_{\infty}^{1}$ and $g_{i}^{2} \rightarrow g_{\infty}^{2}$. To show that $G_{\infty}$ has inverses, let $g_{\infty} \in G_{\infty}$. Let $g_{\infty}^{-1}$ be the limit of the sequence $g_{i}^{-1}$, where $g_{i} \rightarrow g_{\infty}$ and $g_{i}^{-1}$ is the inverse of $g_{i}$ in $G_{i}$. It remains to show that $G_{\infty}$ is closed, so let $g_{\infty}^{n}$ be a sequence in $G_{\infty}$ converging to $g_{\infty}^{\infty} \in G$. We must show that $g_{\infty}^{\infty} \in G_{\infty}$. For each $g_{\infty}^{n}$, find a convergent sequence of $g_{i}^{n}$ converging to $g_{\infty}^{n}$, which we know exists by the definition of $G_{\infty}$. For each $n, g_{i}^{n}$ converges to $g_{\infty}^{n}$, and so we can choose $i(n)$ so that $d\left(g_{m}^{n}, g_{\infty}^{n}\right)<1 / n$ for all $m \geq i(n)$. We will now show that $g_{i(n)}^{n} \rightarrow g_{\infty}^{\infty}$. Let $\varepsilon>0$. Fix $n_{o}$ large enough so that $\varepsilon-1 / n_{o}>0$. Now choose $n_{1}$ so that for all $n \geq n_{1}, d\left(g_{\infty}^{\infty}, g_{\infty}^{n}\right)<\varepsilon-1 / n_{o}$, as $g_{\infty}^{n} \rightarrow g_{\infty}^{\infty}$. Let $N$ be the maximum of $n_{o}$ and $n_{1}$. Given $n \geq N$, we see that $d\left(g_{\infty}^{\infty}, g_{i(n)}^{n}\right) \leq d\left(g_{\infty}^{\infty}, g_{\infty}^{n}\right)+d\left(g_{\infty}^{n}, g_{i(n)}^{n}\right) \leq$ $\varepsilon-1 / n_{o}+1 / n \leq \varepsilon$, as desired. Now, $G_{\infty}$ is a closed group in $G$, and so we know it is compact.

Notice that at this point, $G_{\infty}$ might only consist of id $\in G$, since there may not be any convergent sequences $g_{i}$. We will next show that by taking a subsequence, we can assume that $G_{\infty}$ has Property (1c), while also proving Property (1b). Property (1c) implies that $G_{\infty}$ is non-trivial since a Lie group does not contain any subgroup within a small neighborhood of the identity. Let $U$ be an open neighborhood of $G_{\infty}$. Assume for contradiction that $G_{\infty}$ does not have Property (1c). Then $\forall i_{o}, \exists i \geq i_{o}$ so that $G_{i} \not \subset U$. Choose a subsequence so that $G_{i} \not \subset U$ and choose $g_{i} \in G_{i}$ so that $g_{i} \notin U$. Now, a subsequence of $g_{i}$ must converge to $g_{\infty}^{1} \in G$ outside of $U$ or on the boundary of $U$. We know that $g_{\infty}^{1} \notin G_{\infty}$, since we assumed $g_{i} \notin U$. Restrict the groups to those $i$ 's that are in the subsequence converging to $g_{\infty}^{1}$. Form $G_{\infty}^{1}$ corresponding to this restricted subsequence. From above, we know that $G_{\infty}^{1}$ is a compact group. To show that $G_{\infty} \subset G_{\infty}^{1}$, let $g_{\infty} \in G_{\infty}$. Find a sequence $g_{i} \rightarrow g_{\infty}$. Look at the subsequence of $g_{i}$ consisting only of the $i$ 's we used to form $G_{\infty}^{1}$. This subsequence must also converge to $g_{\infty}$, which is thus in $G_{\infty}^{1}$, by definition. Recall that $g_{\infty}^{1}$ is not in $G_{\infty}$, but it is in $G_{\infty}^{1}$, and so $G_{\infty}$ is a proper subgroup of $G_{\infty}^{1}$. If $G_{\infty}^{1}$ does not satisfy Property (1c), then repeat the process. If this process continues on indefinitely, then we obtain a sequence of proper subgroups $G_{\infty} \subset G_{\infty}^{1} \subset G_{\infty}^{2} \subset \cdots G_{\infty}^{n} \subset \cdots$, which are compact and contained in $G$.

Now $\left(G^{n}\right)^{o}$, the identity component of $G_{\infty}^{n}$, must also form a sequence of subgroups all contained in $G$. Look at the Lie algebras $\mathfrak{g}^{n}$ of $\left(G^{n}\right)^{o}$. They must form a sequence of subalgebras. These are all in $\mathfrak{g}$, the Lie algebra of $G$, which is finite dimensional, so eventually they must all have the same dimension. Now $\left(G^{n}\right)^{o}$ form a connected sequence of subgroups, and eventually they must all have the same Lie algebra dimension. Hence, they must eventually be the same Lie group. Without loss of generality, we can assume that we have a sequence of 
proper subgroups $G_{\infty} \subset G_{\infty}^{1} \subset G_{\infty}^{2} \subset \cdots G_{\infty}^{n} \subset \cdots$ which are compact, have the same dimension, and have the same identity component $G_{O}$.

Notice that when we choose a subsequence, then the limiting group can only get larger. Also, among all possible limiting groups, there is a largest possible dimension, but in general, one can achieve different limiting groups by choosing different subsequences. We will argue that there exists a limiting group $H_{\infty}$, of largest possible dimension, such that no matter what further subsequence one chooses, the limiting group does not get any larger. If not, one gets a sequence of increasing limiting groups $H_{\infty}^{1}, H_{\infty}^{2}, \ldots$ (each one coming from a decreasing choice of a subsequences), all of which have the same dimension and hence the same id component, but more and more components. Now one can choose a diagonal subsequence of the choice of subsequences, and the corresponding limiting group will contain all $H_{\infty}^{i}$ and hence have infinitely many components. But this cannot be since the limiting group is a closed subgroup of $G$, a compact Lie group, which always has only finitely many components. Hence, there exists a limiting group $H_{\infty}$ which satisfies Property (1b). In addition, since the negation of Property (1c) above resulted in the formation of larger limiting groups by taking subsequences, $H_{\infty}$ must also satisfy Property (1c).

Relabel $G_{i}$ to the restricted subsequence of groups used to converge to $H_{\infty}$ as above and in definition (1a). Relabel so that $G_{\infty}=H_{\infty}$. Now, we know that $G_{i} \rightarrow G_{\infty}$.

Proof of (2). We can now apply a Theorem of Montgomery and Zippin [28] which says if $G$ is a Lie group and $K$ is a compact subgroup of $G$, then there exists in $G$ an open set $U$ containing $K$, with the property that for each subgroup $H$ of $G$ lying in $U$, there is an element $g$ of $G$ such that $g^{-1} H g \subset K$. Applying this to our case, let $G$ be $G$ and let $K$ be $G_{\infty}$, as above. Choose $U$ as in MontgomeryZippin. Choose $i_{o}$ as in Property 1c. Relabel our $G_{i}$ so that they begin at $i_{o}$, with $G_{1}=G_{i_{o}}, \ldots$ We have restricted to the tail of our original group sequence, so this will not change our definition of $G_{\infty}$ as in definition (1a). Apply MontgomeryZippin to choose $g(i) \in G$ so that $g(i) G_{i} g(i)^{-1} \subset G_{\infty}$. Look at $g(i)$. This is a sequence in the compact Lie group $G$, so there is a subsequence which converges. Restrict the $i$ 's further so that $g(i) \rightarrow h \in G$. To show that $h \in N_{G}\left(G_{\infty}\right)$, notice that for any $g_{\infty} \in G_{\infty}$, we can find $g_{i} \rightarrow g_{\infty}$. Restrict the $g_{i}$ 's to the $i$ 's we are now using. Then $g_{i}$ still converges to $g_{\infty}$, since we have taken a subsequence of a converging sequence. Hence, $\boldsymbol{g}(i) g_{i} g(i)^{-1} \in G_{\infty}$, since $g(i) G_{i} g(i)^{-1} \subset G_{\infty}$. Taking limits, we see that $h g_{\infty} h^{-1} \in G_{\infty}$, since $G_{\infty}$ is closed, and so $h \in N_{G}\left(G_{\infty}\right)$. We claim that $g(i) G_{i} g(i)^{-1} \rightarrow G_{\infty}$ is also true. Given $g_{\infty} \in G_{\infty}$, we will show that there is some conjugate sequence converging to $g_{\infty}$. Look at $h^{-1} g_{\infty} h$, which is an element of $G_{\infty}$ since $h \in N_{G}\left(G_{\infty}\right)$, so call it $g_{\infty}^{\prime}$. Hence, we can choose $g_{i} \in G_{i}$ so that $g_{i} \rightarrow g_{\infty}^{\prime}$, since $G_{i} \rightarrow G_{\infty}$. Restrict this sequence to the $i$ 's we now have. Then, $g(i) g_{i} g(i)^{-1} \rightarrow h g_{\infty}^{\prime} h^{-1}=h\left(h^{-1} g_{\infty} h\right) h^{-1}=g_{\infty}$. Hence $g(i) G_{i} g(i)^{-1} \rightarrow G_{\infty}$. Since they are already chosen as subgroups of $G_{\infty}$, we know that $g(i) G_{i} g(i)^{-1} \hookrightarrow G_{\infty}$, as desired. 
Remark 4.6. If $G_{i} \rightarrow G_{\infty}$, where $G_{\infty}$ is a closed group, then if we restrict the sequence, $G_{i_{k}} \rightarrow G_{\infty}$.

Lemma 4.7. Let $G_{i} \hookrightarrow G_{\infty}$. Then there is a subsequence and a connected normal compact subgroup $K$ of $G_{\infty}^{o}$ so that $G_{i}^{o} \rightarrow K$. There exists a further subsequence so that $G_{i}$ is conjugate to $G_{i}^{\prime}$ within $G_{\infty}$, where $G_{i}^{\prime} \hookrightarrow G_{\infty}$ and $\left(G_{i}^{\prime}\right)^{o} \hookrightarrow K$.

Proof. Assume that $G_{i} \rightarrow G_{\infty}$. Now $G_{i}^{o}$ are a sequence of closed subgroups of $G$, so apply the proof of part (a) from Lemma 4.5 to obtain a group $K$ so that $G_{i}^{o} \rightarrow K$. To see that $K$ is normal in $G_{\infty}$, let $g_{\infty} \in G_{\infty}$ and $k \in K$. Choose $g_{i} \in G_{i}$ so that $g_{i} \rightarrow g_{\infty}$, as in the definition of $G_{\infty}$. Choose $g_{i}^{o} \in G_{i}^{o}$, as in the definition of $K$, so that $g_{i}^{o} \rightarrow k$. Notice that $G_{i}^{o} \triangleleft G_{i}$ and so $g_{i} g_{i}^{o} g_{i}^{-1} \subset G_{i}^{o}$. By taking limits, we see that $g_{\infty} \mathrm{kg}_{\infty}^{-1} \subset K$. Therefore, $K \triangleleft G_{\infty}$.

To show that by taking a subsequence, $G_{i}$ is conjugate within $G_{\infty}$ to $G_{i}^{\prime}$ with $G_{i}^{\prime o} \hookrightarrow K$, notice that $G_{\infty}$ is a compact subgroup of $G$, and so it is a Lie group. In addition, $G_{i}^{o} \subset G_{\infty}$ since $G_{i} \hookrightarrow G_{\infty}$. We know that $G_{i}^{o} \rightarrow K$, so apply Lemma 4.5 to $G_{i}^{o} \rightarrow K$, within the Lie group $G_{\infty}$. Therefore, we can find $g(i) \in G_{\infty}$ so that $g(i) G_{i}^{o} g(i)^{-1} \hookrightarrow K$.

To show that $K$ is a connected subgroup of $G_{\infty}^{o}$, notice that conjugation preserves components, so the conjugates of $G_{i}^{o}$ are connected. Since they are subgroups of $K$, they must be contained within $K^{o}$. Yet, the components of $K$ are separated, so the conjugates must converge within $K^{o}$. Hence $K=K^{o}$. In addition, $G_{i}^{o} \subset G_{\infty}^{o}$, since $G_{i} \subset G_{\infty}$. Then $K \subset G_{\infty}^{o}$ since $G_{\infty}^{o}$ is closed. Since $G_{\infty}^{o} \subset G_{\infty}$ and $K \triangleleft G_{\infty}$, we see that $K \triangleleft G_{\infty}^{o}$, as desired.

To show that $g(i) G_{i} g(i)^{-1} \hookrightarrow G_{\infty}$, notice that $g(i) G_{i} g(i)^{-1} \subset G_{\infty}$ since $G_{i} \subset G_{\infty}$, by definition of $G_{i} \leftrightarrow G_{\infty}$, and $g(i) \in G_{\infty}$ as above. To show that $g(i) G_{i} g(i)^{-1} \rightarrow G_{\infty}$, notice that even though we have restricted the $i$ 's used in the definition of $G_{\infty}$, we still have $G_{i} \rightarrow G_{\infty}$ by the remark at the end of the proof of Lemma 4.5. Thus, by a similar argument in the proof of Lemma 4.5(1b), we see that $g(i) G_{i} g(i)^{-1} \rightarrow G_{\infty}$.

Lemma 4.8. ([3]) There are only finitely many semi-simple subalgebras of a compact semi-simple Lie algebra, up to conjugacy.

Proof. Let $A$ be the set of $k$-dimensional subalgebras of a compact semisimple Lie algebra $\mathfrak{g}$. Let $\mathfrak{h} \subset \mathfrak{g}$ be semi-simple, and assume that the dimension of $\mathfrak{h}$ is $k$. Let $B_{\mathfrak{h}}$ be the set of all subalgebras in $A$ conjugate to $\mathfrak{h}$. We will show that $B_{\mathfrak{h}}$ is open and closed in $A$ and hence a component of $A$.

To show that $B_{\mathfrak{h}}$ is open, let $\mathfrak{h}_{1} \in B_{\mathfrak{h}}$. We'll show that there is a neighborhood $U$ of $\mathfrak{h}_{1}$ so that $\mathfrak{h}_{2} \subset U \rightarrow \mathfrak{h}_{2}$ is conjugate to $\mathfrak{h}_{1}$. Let $H_{1}$ and $H_{2}$ be the corresponding connected subgroups of the corresponding Lie group $G$. Notice that $Q_{i}=-B_{\mathfrak{g}_{\mid \mathfrak{h}_{i}}}$ is invariant under $\operatorname{Ad}\left(H_{i}\right)$ and so it extends to a bi-invariant metric on $H_{i}$, which we again call $Q_{i}$. We will now show that the diameter of $H_{i}$ with bi-invariant metric $Q_{i}$ is bounded independently of $i$. 
If $Q$ is a bi-invariant metric on $G$, then $\operatorname{Ric}_{Q}(x, y)=-\frac{1}{4} B_{\mathfrak{g}}$. To see this, note that by [13, p. 103] $R(x, y) z=\frac{1}{4}[[x, y], z]$ for any Lie group with a bi-invariant metric. Therefore,

$$
\begin{aligned}
\operatorname{Ric}_{Q}(x, y) & =\operatorname{tr}(z \rightarrow R(x, z) y)=\operatorname{tr}\left(z \rightarrow \frac{1}{4}[[x, z], y]\right) \\
& =\operatorname{tr}\left(z \rightarrow-\frac{1}{4}[[z, x], y]\right)=\operatorname{tr}\left(z \rightarrow-\frac{1}{4}[y,[x, z]]\right) \\
& =-\frac{1}{4} \operatorname{trad} y \circ \operatorname{ad} x=-\frac{1}{4} B(y, x)=-\frac{1}{4} B(x, y),
\end{aligned}
$$

as desired.

Notice that $B_{\mathfrak{h}_{i}}=\lambda_{i} B_{\left.\mathfrak{g}\right|_{\mathfrak{h} i}}$ for some $\lambda_{i}$, and so

$$
\operatorname{Ric}_{Q_{i}}=-\frac{1}{4} B_{\mathfrak{h}_{i}}=-\frac{1}{4} \lambda_{i} B_{\left.\mathfrak{g}\right|_{\mathfrak{h}_{i}}}=\frac{1}{4} \lambda_{i} Q_{i} .
$$

Applying Bonnet-Myers, we see that $\operatorname{diam}\left(H_{i}^{o}, Q_{i}\right) \leq 2 \pi / \sqrt{\lambda_{i}}$. Now, the exponential map maps $\left\{X \in \mathfrak{g} \mid Q_{i}(X, X) \leq 2 \pi / \sqrt{\lambda_{i}}\right\}$ onto $H_{i}$. This implies that if $\mathfrak{h}_{1}$ is close to $\mathfrak{h}_{2}$, then $H_{1}$ is close to $H_{2}$. We apply Montgomery-Zippin to $H_{1}$ and $H_{2}$ within the compact Lie group $G$ to obtain conjugacy of $H_{1}$ and $H_{2}$. Hence we have conjugacy of the subalgebras via the exponential map.

To show that $B_{\mathfrak{h}}$ is closed, let $\mathfrak{h}_{i}$ be an infinite sequence of subalgebras in $B_{\mathfrak{h}}$. Now, $\mathfrak{h}_{i}=\operatorname{Ad}\left(g_{i}\right) \mathfrak{h}$, for $g_{i} \in G$, where $g_{i} \rightarrow g_{\infty}$ for some $g_{\infty} \in G$ by compactness of $G$. Hence, $\mathfrak{h}_{i} \rightarrow \operatorname{Ad}\left(g_{\infty}\right) \mathfrak{h} \in B_{\mathfrak{h}}$, as desired.

To show that $B_{\mathfrak{h}}$ is closed, let $\mathfrak{h}_{1} \in B_{\mathfrak{h}}$.

Now $B_{\mathfrak{h}}$ is a component of $A$, which has only finitely many components by compactness, and so we obtain the desired result.

Lemma 4.9. Let $G_{i} \hookrightarrow G_{\infty}$ and $G_{i}^{o} \hookrightarrow K$. Then there exists a subsequence so that the Lie algebras of $G_{i}^{o}$ converge as subspaces to a subalgebra of the Lie algebra of $K$.

(a) If the Lie algebra of $K$ is non-trivial and semi-simple, then we can find a subsequence so that $G_{i}^{o}=K$.

(b) If $G_{i}^{o}$ is semi-simple, then $K$ is semi-simple.

(c) If $K$ is not abelian or semi-simple, then we can find a subsequence, up to conjugacy, such that the semi-simple part of $G_{i}^{o}$ is equal to the semi-simple part of $K$ and $Z\left(G_{i}^{o}\right)^{o} \subset Z(K)$.

Proof. We know that $K$ is connected, as in Lemma 4.7, so consider the Lie algebras $\mathfrak{g}_{i}$ and $\mathfrak{K}$, all inside of the vector space $V$. We will show that $\mathfrak{g}_{i}$ converge as subspaces to a subalgebra of $\mathfrak{K}$. Since $G_{i}^{o} \subset K$, we know that $\mathfrak{g}_{i}$ is a subalgebra of $\mathfrak{K}$. We can assume that the $\mathfrak{g}_{i}$ all have the same dimension $m$. The set of all $m$ dimensional subspaces in $V$ is compact, so choose a converging subsequence and rename it $\mathfrak{g}_{i}$. Now $\mathfrak{g}_{i}$ converge to a subspace, call it $\mathfrak{s}$, of dimension $m$. To show that $\mathfrak{s}$ is an algebra, let $s_{1}, s_{2} \in \mathfrak{s}$. Pick $x_{i}, y_{i} \in \mathfrak{g}_{i}$ so that $x_{i} \rightarrow s_{1}$ and $y_{i} \rightarrow s_{2}$. Now $\left[x_{i}, y_{i}\right] \in \mathfrak{g}_{i} \subset \mathfrak{K}$. Taking limits, we see that $\left[s_{1}, s_{2}\right] \in \mathfrak{s}$, as desired. and $\mathfrak{s}$ is a subalgebra of $\mathfrak{K}$. Define $S=\exp \mathfrak{s}$. Then $S$ is a connected subgroup of $K$. 
Proof of (a). Assume that $\mathfrak{K}$ is semi-simple. If $\mathfrak{g}_{i}$ is not semi-simple for arbitrarily large $i$, then it must have a non-trivial center. Look on the group level and restrict to these $i$ 's. By Lemma 4.5, we can assume that $Z\left(G_{i}^{o}\right)^{o} \rightarrow L$. We still know that $G_{i}^{o} \hookrightarrow K$, by the remark at the end of the proof of Lemma 4.5. Since elements in the center will commute with all other group elements in each $G_{i}^{o}$, then in the limit, these elements will commute with all other group elements in $K$ and hence converge to elements in $Z(K)$. Therefore, $L \subset Z(K)$. In addition, by Lemma 4.5, we can assume that $Z\left(G_{i}^{o}\right)^{o}$ are conjugate to a subgroup of $Z(K)$. Yet, we assumed that $\mathfrak{K}$ was simple, and we know that $K$ is connected. Hence, the center of $K$ must be finite. Hence $Z\left(G_{i}^{o}\right)^{o}$ are conjugate to subgroups of a connected finite group and therefore must be trivial. Hence, $\mathfrak{Z}\left(\mathfrak{g}_{i}\right)$ must be trivial, a contradiction to our assumption.

Therefore, $\mathfrak{g}_{i}$ must be semi-simple. We have already assumed that the $\mathfrak{g}_{i}$ all have the same dimension $m$. Look at $\mathfrak{g}_{i}=\mathfrak{g}_{i}^{1}+\mathfrak{g}_{i}^{2}+\cdots+\mathfrak{g}_{i}^{q}$, where each factor is a simple ideal. By restricting to a subsequence, we can assume that each $\mathfrak{g}_{i}$ has exactly $q$ simple ideals. We know that $B_{\mathfrak{g}_{i} \mathfrak{g}_{i}^{p}}=\lambda_{i}^{p} B_{\mathfrak{K}_{\mid \mathfrak{g}_{i}^{p}}}$ for each $p$. The $\mathfrak{g}_{i}^{p}$ are all subalgebras of $\mathfrak{K}$, which is also semi-simple. Since they are simple, they are certainly semi-simple. There are only finitely many semi-simple subalgebras of a semi-simple Lie algebra, up to conjugacy, by Lemma 4.8. Since the Killing form is preserved under conjugacy, then there are only finitely many values for $\lambda_{i}^{p}$. They are each non-zero since $\mathfrak{g}_{i}^{p}$ is simple, so choose the smallest $\lambda_{i}$ and call it $\lambda$. We know that $\lambda$ is not zero.

Notice that $Q_{i}=-B_{\mathfrak{K}_{\mid g_{i}}}$ is invariant under $\operatorname{Ad}\left(G_{i}^{o}\right)$ and so it extends to a bi-invariant metric on $G_{i}^{o}$, which we again call $Q_{i}$. We will now show that the diameter of $G_{i}^{o}$ with bi-invariant metric $Q_{i}$ is bounded by a constant independent of $i$. We know that

$$
\operatorname{Ric}_{Q_{i}}=-\frac{1}{4} B_{\mathfrak{g}_{i}} \geq-\frac{1}{4} \lambda B_{\mathfrak{K}_{\mathfrak{g}_{i}}}=\frac{1}{4} \lambda Q_{i} .
$$

Applying Bonnet-Myers, we see that $\operatorname{diam}\left(G_{i}^{o}, Q_{i}\right) \leq 2 \pi / \sqrt{\lambda}$.

Let $g_{\infty}^{o} \in K$ and choose $g_{i}^{o} \in G_{i}^{o}$ with $\lim g_{i}^{o}=g_{\infty}^{o}$. Choose $x_{i} \in \mathfrak{g}_{i}$ with $\left|x_{i}\right| \leq 2 \pi / \sqrt{\lambda}$ so that $g_{i}^{o}=\exp x_{i}$. Hence, $x_{i}$ will converge to $x_{\infty} \in \mathfrak{s}$. Since $\exp$ is continuous, we know that $g_{\infty}^{o}=\exp x_{\infty} \in S$. Hence $K=S$. Now the connected groups $G_{i}^{o}$ are subgroups of $K$ with the same Lie algebra dimension, so they must be the same Lie group. Hence, $G_{i}^{o}=K$.

Proof of $(\mathrm{b})$. Assume that $G_{i}^{o}$ is semi-simple. Assume for contradiction that $K$ is not semi-simple. Then $\mathfrak{K}=\mathfrak{K}^{s s}+\mathfrak{Z}(\mathfrak{K})$.

Look at the projection $\pi: \mathfrak{K}^{s s} \oplus \mathfrak{Z}(\mathfrak{K}) \rightarrow \mathfrak{Z}(\mathfrak{K})$, which is a homomorphism of Lie algebras. Now $\pi_{\mid \mathfrak{g}_{i}}: \mathfrak{g}_{i} \rightarrow \mathfrak{Z}(\mathfrak{K})$ is also a homomorphism, and so $\mathfrak{a}_{i}=$ $\operatorname{ker}\left(\boldsymbol{\pi}_{\mid \mathfrak{g}_{i}}\right)$, the kernel, is an ideal in $\mathfrak{g}_{i}$. In addition, $\mathfrak{g}_{i}$ is semi-simple, and so there exists an ideal $\mathfrak{b}_{i}$ so that $\mathfrak{g}_{i}=\mathfrak{a}_{i} \oplus \mathfrak{b}_{i}$. We know that $\mathfrak{b}_{i}$ is semi-simple since $\mathfrak{g}_{i}$ is semi-simple. 
Then $\pi_{\mid \mathfrak{g}_{i}}: \mathfrak{g}_{i} / \operatorname{ker}\left(\boldsymbol{\pi}_{\mid \mathfrak{g}_{i}}\right) \rightarrow \mathfrak{Z}(\mathfrak{K})$, and so $\pi_{\mathfrak{g}_{i}}: \mathfrak{b}_{i} \rightarrow \mathfrak{Z}(\mathfrak{K})$ is one-to-one. Therefore $\mathfrak{b}_{i}$ is isomorphic to a subalgebra of $\mathfrak{Z}(\mathfrak{K})$. We have arrived at a contradiction unless $\mathfrak{b}_{i}=0$, since $\mathfrak{b}_{i}$ is semi-simple. Hence $\mathfrak{g}_{i} \subset \mathfrak{K}^{s s}$. Now, repeat the proof of (a) to see that $G_{i}^{o}=K^{s s}$. Hence $K^{s s}=K$, by definition of convergence of $G_{i}^{o}$ to $K$. Thus, $K$ is semi-simple, as desired.

Proof of (c). Assume that $K$ is not abelian. As in the proof of (a), we know that there exists a subsequence so that $Z\left(G_{i}^{o}\right)^{o} \rightarrow L \subset Z(K)$. Up to conjugacy within $K$ and by taking a further subsequence, we may assume that $Z\left(G_{i}^{o}\right)^{o} \hookrightarrow L \subset Z(K)$, $G_{i}^{o} \hookrightarrow K$, and $G_{i} \hookrightarrow G_{\infty}$. This holds by arguments similar to Lemma 4.7, using the fact that $Z\left(k G_{i}^{o} k^{-1}\right)^{o}=k Z\left(G_{i}^{o}\right)^{o} k^{-1}$ for $k \in K$, which holds as conjugation preserves components and the center.

Notice that if $G_{i}$ were abelian for arbitrarily large $i$, then restricting to those groups, $G_{i} \hookrightarrow K$, and so $K$ would have to be abelian, a contradiction. Hence, we know that there is an $i_{o}$ so that $\mathfrak{g}_{i}^{s \mathcal{S}}$ is non-trivial for $i \geq i_{o}$. Restricting to these $i$ 's, we have that $\left(G_{i}^{o}\right)^{s S} \neq 0$.

Define $N$ as the compact group so that $\left(G_{i}^{o}\right)^{s s} \rightarrow N$. Up to conjugacy within $K$ and by taking a further subsequence, we may assume that $\left(G_{i}^{o}\right)^{\mathcal{S S}} \hookrightarrow N$, as conjugation preserves the semi-simple factor. Also, $G_{i}^{o} \hookrightarrow K$, and $G_{i} \hookrightarrow G_{\infty}$, as before. In addition, this does not affect $Z\left(G_{i}^{o}\right)^{o} \hookrightarrow L$. Notice that $Z\left(G_{i}^{o}\right)^{o} \hookrightarrow$ $L \subset Z(K)$ implies $Z\left(G_{i}^{o}\right)^{o} \subset Z(K)$, and so conjugation by an element of $K$ is the identity on $Z\left(G_{i}^{o}\right)^{o}$. Hence, we still have that $Z\left(G_{i}^{o}\right)^{o} \rightarrow L \subset Z(K)$.

Apply part (b) to show that $N$ is semi-simple. Now $N=\left(G_{i}^{o}\right)^{s s}$ by part (a).

We will show that we can write $G_{i}^{o}$ as $N \cdot Z\left(G_{i}^{o}\right)^{o}$, so let $g_{i}^{o} \in G_{i}^{o}$. First notice that both $N=\left(G_{i}^{o}\right)^{S S}$ and $Z\left(G_{i}^{o}\right)^{o}$ are connected subgroups of $G_{i}^{o}$. Look on the Lie algebra level. Then $\mathfrak{g}_{i}=\left(\mathfrak{g}_{i}^{o}\right)^{s s}+\mathfrak{Z}\left(\mathfrak{g}_{i}^{o}\right)$. Since $G_{i}^{o}$ is compact, we can find $x \in \mathfrak{g}_{i}, x_{s s} \in\left(\mathfrak{g}_{i}^{o}\right)^{s s}$, and $x_{\mathfrak{Z}} \in \mathfrak{Z}\left(\mathfrak{g}_{i}^{o}\right)$ so that $g_{i}^{o}=\exp (t x)=\exp \left(t x_{s s}+\right.$ $\left.t x_{\mathfrak{Z}}\right)$, where $x=x_{s s}+x_{\mathfrak{Z}}$. Since $\left[x_{s s}, x_{\mathfrak{Z}}\right]=0$, as $x_{\mathfrak{Z}} \in \mathfrak{Z}\left(\mathfrak{g}_{i}^{o}\right)$, we know that $g_{i}^{o}=\exp \left(t x_{s s}+t x_{\mathfrak{Z}}\right)=\exp \left(t x_{s s}\right) \cdot \exp \left(t x_{\mathfrak{Z}}\right)$. Thus, $g_{i}^{o}=n_{i} \cdot z_{i}$, for some $n_{i} \cdot z_{i} \in N \cdot Z\left(G_{i}^{o}\right)^{o}$, as desired.

To show that $\left(G_{i}^{o}\right)^{s s}=K^{s s}$, let $g_{i}^{o} \in G_{i}^{o}$. Then $g_{i}=n_{i} \cdot z_{i} \in N \cdot Z\left(G_{i}^{o}\right)^{o}$. Taking limits, we see that $G_{i}^{o} \rightarrow N \cdot Z(K)$, since $Z\left(G_{i}^{o}\right)^{o} \hookrightarrow Z(K)$. Yet $G_{i}^{o} \hookrightarrow K=$ $K^{s s} \cdot Z(K)$. Hence, $N=K^{s s}$, and so $\left(G_{i}^{o}\right)^{s s}=K^{s s}$, as $N=\left(G_{i}^{o}\right)^{s s}$.

Lemma 4.10. If $G$ is transitive on $M$, a connected manifold, then $G^{o}$ is also transitive on $M$.

Proof. Let $G^{o}$ be the connected component of the identity. By a standard argument, we will show that any orbit is both open and closed. Notice that $G_{\infty}^{o}$ is compact and hence the orbit is compact and hence closed. To show that an orbit is open, pick a point $p$ in the orbit of $G_{\infty}^{o}$. In $M$, we can find a neighborhood around this point which stays within the orbit. Intersect this neighborhood with a neighborhood of the identity. Since $p$ is in the orbit of $G_{\infty}^{o}$, we know this is nonempty. Hence any orbit is open. Since $M$ is connected, we know that the orbit 
must be everything or empty. We know the orbit is not empty, so it is everything. Therefore, $S^{n} / G_{\infty}^{o}$ is transitive.

Lemma 4.11. The following is the classification of connected transitive orthogonal subgroups and the spheres on which they act [2]:

(1) $S O(n+1)$ on $S^{n}$

(2) $U(n) \subset S O(2 n)$ on $S^{2 n-1}$

(3) $S U(n) \subset S O(2 n)$ on $S^{2 n-1}$

(4) $S p(n) \times S p(1) \subset S O(4 n)$ on $S^{4 n-1}$

(5) $S p(n) \times U(1) \subset S p(n) \times S p(1)$ on $S^{4 n-1}$

(6) $S p(n) \subset S p(n) \times S p(1) \subset S O(4 n)$ on $S^{4 n-1}$

(7) $\operatorname{Spin}(9) \subset S O(16)$ on $S^{15}=\operatorname{Spin}(9) / \operatorname{Spin}(7)$

(8) $\operatorname{Spin}(7) \subset S O(8)$ on $S^{7}=\operatorname{Spin}(7) / G_{2}$

(9) $G_{2} \subset S O(7)$ on $S^{6}=G_{2} / S U(3)$

Theorem 4.3. If $G$ is a non-transitive group, then there exists $\varepsilon$, depending only on $n$, so that $\operatorname{diam}\left(S^{n} / G\right) \geq \varepsilon(n)$.

Proof. Fix $n$, and assume for contradiction that we have a sequence of closed, non-transitive $G_{i} \subset O(n+1)$ so that $\operatorname{diam}\left(S^{n} / G_{i}\right) \rightarrow 0$.

We know that conjugation does not change the diameter of the resulting quotient. Therefore, changing the sequence via restriction and conjugation, will not change that $\operatorname{diam}\left(S^{n} / G_{i}\right) \rightarrow 0$. So, without loss of generality, we may define $G_{\infty}$ as in Lemma 4.5, and we may assume that $G_{i} \hookrightarrow G_{\infty}$ and $G_{i}^{o} \hookrightarrow K$ by Lemmas 4.5 and 4.7 .

Now $\operatorname{diam}\left(S^{n} / G_{\infty}\right)=0$ since a group has smaller resulting diameter when compared with the resulting diameter of its subgroups. Since $G_{\infty}$ is closed, then $G_{\infty}$ must be transitive on $S^{n}$, as desired.

If $K=\mathrm{Id}$, then $G_{i}^{o}=\mathrm{Id}$. Hence, $G_{i}$ is finite. Yet, the diameter of $S^{n} / G_{i} \rightarrow$ 0 . This contradicts Theorem 3.14 which produces a lower bound, $\varepsilon(n)$, on the diameter resulting from finite groups. Hence $K \neq \mathrm{Id}$ and $G_{i}^{o} \neq \mathrm{Id}$.

Case 1: $G_{\infty}^{o}$ is simple. We know that $K$ is connected and normal in $G_{\infty}^{o}$. In addition, $G_{\infty}^{o}$ is simple and $K \neq \mathrm{Id}$. Hence, we know that $K=G_{\infty}^{o}$. Hence $K$ is transitive and simple. Apply Lemma 4.9 to see that $G_{i}^{o}=K$. Since $K$ is transitive, we see that $G_{i}^{o}$ is transitive, and so we have arrived at a contradiction.

Case 2: $G_{\infty}^{o}$ is not simple. Since $G_{\infty}^{o}$ is not simple, (2), (5), and (4) in Lemma 4.11 are the only groups which remain to be examined.

Converting the notation in Lemma 4.11 to our notation in $O(n+1)$ acting on $S^{n}$, we see that $G_{\infty}^{o}$ is not simple only when $n+1$ is even. Let $n+1$ be even. Let $j=(n+1) / 2$. Then $G_{\infty}^{o}$ is one of the following,

(a) $U(j) \subset S O(n+1)$,

(b) $S p(j / 2) \times S p(1) \subset S O(n+1)$, if $j$ is even,

(c) $S p(j / 2) \times U(1) \subset S O(n+1)$, if $j$ is even. 
where (b) and (c) only occur if $n+1$ is divisible by 4 .

We will first examine these actions. In (a), write $\mathbb{R}^{n+1}=\mathbb{C}^{j}$. Then $A \in U(j)$ : $v \rightarrow A v$, and $z \in U(1)=Z(U(j)): v \rightarrow z v$. In (b), examine $\mathbb{R}^{n+1}=\mathbb{q}^{j / 2}$. Then $A \in S p(j / 2)$ and $q \in S p(1)$ acts via $v \rightarrow A(v) q$. In (c), $A \in S p(j / 2)$ and $z \in U(1) \subset S p(1)$ acts via $v \rightarrow A(v) z$.

We know that $K$ is connected, non-trivial, and a normal subgroup in $G_{\infty}^{o}$, and that $G_{i}^{o}$ are subgroups of $K$ that converge to $K$.

We will now more closely examine the actions. In (a), the only proper, connected, normal subgroups are $U(1)$, the Hopf action, and $S U(j)$, which acts transitively. In (b), the only proper, connected, normal subgroups are $S p(j / 2)$, which acts transitively, and $S p(1)$. In (c), the only proper, connected normal subgroups are $S p(j / 2)$, which acts transitively, and $U(1)$.

Hence, we see that $K$ must be $G_{\infty}^{o}$ itself, or $S U(j), U(1), S p(j / 2)$, or $S p(1)$. If $K=S U(j)$ or $S p(j / 2)$, then $K$ is simple and transitive, so apply arguments in Case 1 to obtain a contradiction. We will examine the remaining groups to obtain a contradication in each case.

Case 2A: $K=U(1)$.

If we are in (a), then the $U(1)$ action on $\mathbb{R}^{n+1}$ is the Hopf action. To further examine the $U(1)$ action in (c), look at $\mathbb{C}^{2 m}=\mathbb{a}^{m}$ via $(a, b) \rightarrow a+j b=$ $v$. To show that the $U(1)$ action is also the Hopf action, notice that $z(a, b)=$ $(z a, z b)=(a z, b z) \rightarrow a z+j(b z)=(a+j b) z=v z$.

We know that $G_{i}^{o} \neq \mathrm{Id}$ is connected and a subgroup of $K=U(1)$. Hence, $G_{i}^{o}$ is $U(1)$. Now,

$$
S^{n} / G_{i}=\frac{S^{n} / G_{i}^{o}}{G_{i} / G_{i}^{o}}=\frac{S^{n} / U(1)}{G_{i} / U(1)}=\frac{\mathbb{C} P^{j-1}}{G_{i} / U(1)}
$$

Using the submersion metric, we see that $G_{i} / U(1)$ is in the isometry group of $\mathbb{C} P^{j}$, since $G_{i} \triangleleft U(1)$ and so orbits get mapped to orbits. We know that $G_{i} / G_{i}^{o}=$ $G_{i} / U(1)$ is finite. Therefore $G_{i} / U(1)$ is a finite subgroup of the isometry group of $\mathbb{C} P^{n}$. Apply Corollary 3.14.1 to obtain a lower bound on the diameter of $\mathbb{C} P^{j-1} /\left(G_{i} / U(1)\right)$. We have arrived at a contradiction to the assumption that the diameter of $S^{n} / G_{i}$ approaches 0 .

Case 2B: $K=S p(1)$.

We know that $G_{i}^{o} \neq \mathrm{Id}$ is connected and a subgroup of $K=S p(1)$. But, the only connected subgroups of $S p(1)$ are great circles in $S^{3}$, which cannot converge to $S^{3}=S p(1)$. Hence, $G_{i}^{o}$ is $S p(1)$. Also,

$$
S^{n} / G_{i}=\frac{S^{n} / G_{i}^{o}}{G_{i} / G_{i}^{o}}=\frac{S^{n} / S p(1)}{G_{i} / S p(1)}=\frac{\llbracket P^{j / 2-1}}{G_{i} / S p(1)} .
$$


The contradiction is obtained in a similar fashion to Case $3 \mathrm{~A}$, using Corollary 3.14.1 to obtain a lower bound on the diameter of $\llbracket P^{j / 2-1} /\left(G_{i} / S p(1)\right)$.

\begin{tabular}{|l|l|c|c|c|}
\hline \multicolumn{5}{|c|}{ Summary of Diameter Results } \\
\hline \hline \multicolumn{1}{|c|}{$\begin{array}{c}\text { Class of Groups or } \\
\text { Actions }\end{array}$} & $\begin{array}{c}\text { Resulting } \\
\text { Spaces }\end{array}$ & $\begin{array}{c}\text { Lower Bound on } \\
\text { Diameter }\end{array}$ & $\begin{array}{c}\text { Dimension } \\
\text { Achieved }\end{array}$ & $\begin{array}{c}\text { Limit as } \\
n \rightarrow \infty\end{array}$ \\
\hline $\begin{array}{l}\text { Reducible Actions } \\
\text { (Grove, Borzellino) }\end{array}$ & $\frac{\pi}{2}$ & $\frac{\pi}{2}$ \\
\hline $\begin{array}{l}\text { Free Actions } \\
\text { (McGowan, 91, } \\
\text { Flach, 92) }\end{array}$ & Manifolds & $\frac{\pi}{9.63}$ & 3 & $\frac{\pi}{2}$ \\
\hline $\begin{array}{l}\text { Dimension 2 Groups } \\
\text { ([20], [15]) }\end{array}$ & $\begin{array}{l}\text { Orbifolds and } \\
\text { Alexandrov }\end{array}$ & $\frac{\pi}{4.82}$ & 2 & $\frac{\pi}{2}$ \\
\hline Coxaces & $\begin{array}{l}\text { Coxeter } \\
\text { Orbifolds }\end{array}$ & $\frac{\pi}{8.10}$ & 3 & 0 \\
\hline Finite Groups & Orbifolds & $\exists \operatorname{explicit} \varepsilon(n)$ & $n$ & $\frac{\pi}{4}$ \\
\hline $\begin{array}{l}\text { Cohomogeneity-1 } \\
\text { Actions } \\
\text { (Hsiang-Lawson, 71) }\end{array}$ & Intervals & $\frac{\pi}{6}$ & 7 and 13 & $n$ \\
\hline $\begin{array}{l}\text { Infinite, Closed, } \\
\text { Non-Transitive Groups }\end{array}$ & Alexandrov & $\exists \varepsilon(n)$ & $n$ & \\
\hline
\end{tabular}

TABLE 2. Summary of Diameter Results

Case 2C: $K=G_{\infty}^{o}$.

$K$ is transitive. Even though $K$ is not simple, we will still be able to apply arguments which resemble those in Case 1.

Case 2C1: $K=S p(j / 2) \times S p(1)$.

Now $K$ is semi-simple, so apply part (a) of Lemma 4.9 to restrict the sequence so that $G_{i}^{o}=K$. Since $K$ is transitive, we see that $G_{i}^{o}$ is transitive, and so we have arrived at a contradiction.

Case 2C2: $K=U(j) \subset S O(n+1)$ or $S p(j / 2) \times U(1)$.

We satisfy the conditions of part (c) of Lemma 4.9, since $K$ is not abelian or semi-simple. By Lemma 4.9 we can restrict the sequence so that up to conjugacy, 
$\left(G_{i}^{o}\right)^{s s}=K^{s s}$. We have arrived at a contradiction since $K^{s s}=S U(j)$ or $S p(j / 2)$, which is transitive.

There are no other cases remaining, so the theorem is proven.

\section{REFERENCES}

[1] M.A. Armstrong, Groups and symmetry, Undergraduate Texts in Mathematics, Springer, Berlin, 1988.

[2] ARTHUR BESSE, Manifolds all of whose geodesics are closed, A Series of Modern Surveys in Mathematics, no. 93, Springer-Verlag, 1978.

[3] C. BOEHM, M. WANG \& W. ZILLER, A variational approach for homogeneous Einstein metrics, in preparation (2000).

[4] Joseph BorZellino, Riemannian geometry of orbifolds, Ph.D. thesis, University of California Los Angeles, 1992.

[5] - Orbifolds of maximal diameter, Indiana Univ. Math. J. 42 (1993), 37-53.

[6] Pinching theorems for teardrops and footballs of revolution, Bull. Austral. Math. Soc 49 (1994), 353-364.

[7] _ Orbifolds with lower Ricci curvature bounds, Proc. Amer. Math. Soc. 125 (1997), 3011-3018.

[8] Yu Burago, M. Gromov \& G. Perelman, A.d. Alexksandrov spaces with curvatures bounded below (translation), Russian Math. Surveys 47 (1992), 1-58.

[9] LeOnard Charlap, Bieberbach Groups and Flat Manifolds, Springer-Verlag, 1986.

[10] ARJeH CohEN, Finite complex reflection groups, Ann. Scient. Ec. Norm. Sup. (1976), 379-436.

[11] H.S.M. Coxeter, Regular Polytopes, Dover, New York, 1973.

[12] M. Demazure, Surfaces de Del Pezzo ii, Lecture Notes in Math. 777 (1980), 23-25.

[13] Manfredo Do Carmo, Riemannian Geometry, Birkhauser, 1993.

[14] Patrick DU Val, Homographies, Quaternions and Rotations, Oxford Mathematical Monographs, 1964.

[15] B. Dunbar, S. Greenwald, J. McGowan \& C. Searle, Diameters of quotients of 3-spheres, in preparation.

[16] William D. Dunbar, Nonfibering spherical 3-orbifolds, Trans. Amer. Math. Society 341 (1994), 121-142.

[17] Kurt Endl \& Uwe MefFert, Impossiball, Copyright.

[18] Nicole FlaCH, Diametre des quotients de spheres, Ph.D. thesis, Lausanne University, 1992.

[19] , Diametre des varietes a courbure sectionelle positive, C.R Acad Sci Paris Ser I Math 318 (1994), 827-830.

[20] SARAH J. GREENWALD, Diameters of spherical Alexandrov spaces and constant curvature one orbifolds, Ph.D. thesis, University of Pennsylvania, 1998.

[21] K. GRove \& S. MARKVORSEN, New extremal problems for the Riemannian recognition program via Alexandrov geometry, J. Amer. Math. Society 8 (1995), 1-28.

[22] L.C. GROVE \& C.T. BENSON, Finite reflection groups, Graduate Texts in Mathematics, no. 99, Springer-Verlag, 1971. 
[23] W. Hsiang \& B. LaWson, Minimal submanifolds of low cohomogeneity, J. Differential Geometry 5 (1971), 1-38.

[24] JAMES HumphreYS, Reflection groups and Coxeter groups, Cambridge Studies in Advanced Mathematics, no. 29, 1990.

[25] JiLl MCGowan, The diameter function on the space of space forms, Ph.D. thesis, University of Maryland, 1991.

[26] _ The diameter function on the space of space forms, Compositio Math 87 (1993), 79-98.

[27] Jose Montesinos, Classical Tessellations and Three-manifolds, Springer-Verlag, 1985.

[28] D. Montgomery \& L. Zippin, A theorem on Lie groups, Bull. Amer. Math. Soc. 48 (1942), 448-452.

[29] JoHn RatCliffe, Foundations of hyperbolic manifolds, Graduate Texts in Mathematics, no. 149, Springer-Verlag, 1994.

[30] Peter ScotT, The geometries of 3-manifolds., Bull. London Math. Soc. 15 (1983), 401-487.

[31] Katsuhiro Shiohama, An introduction to the geometry of Alexandrov spaces, Lecture Notes Series, Seoul National University, 1993.

[32] W. THURSTON, The geometry and topology of 3-manifolds, (1978).

[33] Whole Systems Design, Masterball.

[34] Joseph Wolf, Spaces of Constant Curvature, Publish or Perish, Inc., 1984.

Department of Mathematics

Appalachian State Univ

Boone, NC 28608

U.S.A.

E-MAIL: sjg@math.appstate.edu

KEY WORDS AND PHRASES: orbifold of constant curvature, Coxeter groups, spherical Alexandrov space, lower bound, quotient spaces

1991 Mathematics Subject Classification: Primary: 53C20

Received: September 8th, 1999. 